\title{
Dynamics of axially accelerating beams with multiple supports
}

\author{
S.M. Bağdatli • E. Özkaya • H.R. Öz
}

Received: 12 March 2013 / Accepted: 14 May 2013 / Published online: 4 June 2013

(C) The Author(s) 2013. This article is published with open access at Springerlink.com

\begin{abstract}
This study represents the transverse vibrations of an axially accelerating Euler-Bernoulli beam resting on multiple simple supports. This is one of the examples of a system experiencing Coriolis acceleration component that renders such systems gyroscopic. A small harmonic variation with a constant mean value for the axial velocity is assumed in the problem. The immovable supports introduce nonlinear terms to the equations of motion due to stretching of neutral axis. The method of multiple scales is directly applied to the equations of motion obtained for the general case. Natural frequency equations are presented for multiple support case. Principal parametric resonances and combination resonances are discussed. Solvability conditions are presented for different cases. Stability analysis is conducted for the solutions; approximate stable and unstable regions are identified. Some numerical examples are presented to
\end{abstract}

S.M. Bağdatli (凶) • E. Özkaya

Department of Mechanical Engineering, Faculty of

Engineering, Celal Bayar University, 45140 Muradiye,

Manisa, Turkey

e-mail: murat.bagdatli@cbu.edu.tr

E. Özkaya

e-mail: erdogan.ozkaya@cbu.edu.tr

H.R. Öz

Department of Genetics and Bioengineering, Faculty of Engineering, Fatih University, 34500, B. Çekmece, İstanbul, Turkey

e-mail: hroz@ fatih.edu.tr show the effects of axial speed, number of supports, and their locations.

Keywords Axially accelerated beam $\cdot$ Multiple support $\cdot$ Perturbation method

\section{Introduction}

Many real-life engineering devices, such as band and chain-saws, conveyor belts, fiber textiles, magnetic tapes, paper sheets, and threadlines, involve vibration of axially accelerating beams. Some practical examples can be modeled as a moving string of thin or thick beams. A vast literature can be found in references $[1,2]$. Transverse vibrations of axially moving strings and beams are investigated by Wickert and Mote [3] including axial tension. Wickert [4] discussed tensioned beams, including nonlinear stretching effects for subcritical and supercritical speed region. Pakdemirli and Ulsoy [5] obtained approximate analytical solutions for variable speed using the method of multiple scales and compared direct-perturbation and discretization-perturbation. Nayfeh et al. [6] showed that direct-perturbation is better for quadratic and cubic nonlinearities. The method of multiple scales and other methods were applied to string-beam transition problem [7-11] for axially accelerating materials. Yurddaş et al. $[12,13]$ investigated nonlinear vibrations of an axially moving string having nonideal 


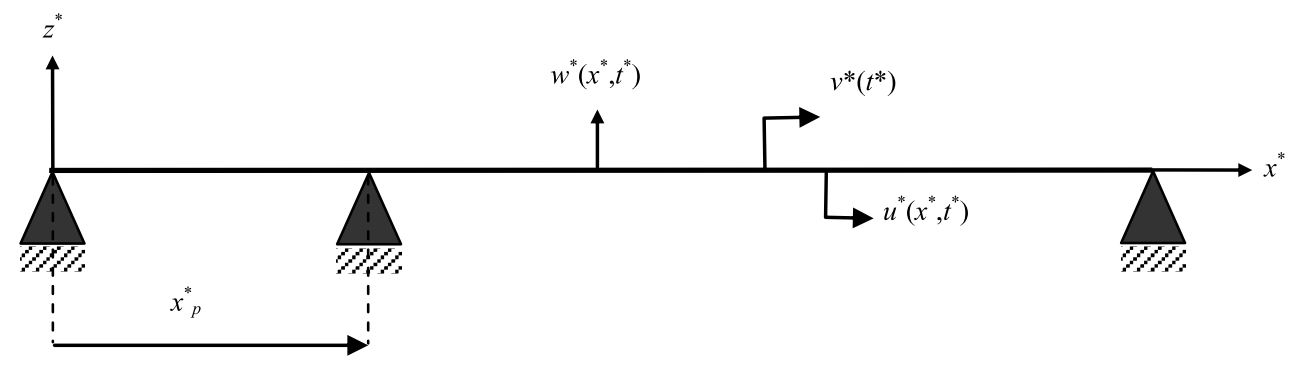

Fig. 1 Axially moving beam on multiple supports

mid-support and multi-support conditions. The variable velocity case for a moving beam was investigated for different end conditions and different resonance cases, including principal parametric and combination types, were discussed in [14-19]. Infinite-mode analysis was performed in [20]. There are also some studies about axially moving beams composed of viscoelastic materials [21-24]. Stationary beams with multiple supports were also investigated in detail. Nonlinear free vibrations of multispan beams on elastic supports were studied by Lewandowski [25], where frequencies and nonlinear mode of vibrations were found by using dynamic stiffness method, and the influence of support flexibility on the frequency amplitude relations was examined. Beams simply supported in span were discussed, and frequency response functions were determined [26, 27]. Nonlinear vibrations and 3:1 internal resonances on multiple supports were investigated, and excitation frequency-frequency response curves were drawn for different support numbers [28, 29]. Bağdatli et al. [30] dynamics of axially accelerating beams with an intermediate support. Tekin et al. [31] investigated three-to-one internal resonances for multi-stepped beam systems. The coupled longitudinal-transverse nonlinear dynamics of an axially accelerating beam was determined [34], and Ghayesh et al. [35] discussed the stability of an axially moving beam supported by an intermediate spring.

In the current manuscript, transverse vibrations of axially moving beams are presented. An EulerBernoulli-type axially moving beam on multiple supports (simply supported) is considered. This type of support may represent contact with multiple boundaries, e.g., cutting a wood or passing through holes. Stretching of the neutral axis introduces a nonlinear effect to the problem. The beam travels with a harmonic axial velocity slightly varying about a constant mean value. The equations of motion are obtained using an energy approach and solved using a perturbation technique. A general support condition in matrix form is presented for multi-support case. Natural frequencies are presented for different flexural rigidity values, support locations, and support numbers. Principal parametric resonances and stability are investigated.

\section{Equations of motion}

Figure 1 shows the axially accelerating beam on multiple supports. $x^{*}, z^{*}$, and $t^{*}$ are spatial and time variables, respectively, $w^{*}$ and $u^{*}$ denote the transverse and axial displacements respectively, and $v^{*}$ is the axial velocity of the beam.

The Lagrangian of the system is given below.

$$
\begin{aligned}
£= & \frac{1}{2} \sum_{m=0}^{n} \int_{x_{m}^{*}}^{x_{m+1}^{*}} \rho A\left\{\left(\dot{w}_{m+1}^{*}+w_{m+1}^{* \prime} v^{*}\right)^{2}\right. \\
& \left.+\left(v^{*}+\dot{u}_{m+1}^{*}+u_{m+1}^{* \prime} v^{*}\right)^{2}\right\} d x^{*} \\
& -\sum_{m=0}^{n}\left[\frac{1}{2} \int_{x_{m}^{*}}^{x_{m+1}^{*}} E A\left(u_{m+1}^{* \prime}+\frac{1}{2} w_{m+1}^{* \prime 2}\right)^{2} d x^{*}\right. \\
& +\frac{1}{2} \int_{x_{m}^{*}}^{x_{m+1}^{*}} E I w_{m+1}^{*^{\prime \prime 2}} d x^{*} \\
& \left.+\int_{x_{m}^{*}}^{x_{m+1}^{*}} P\left(u_{m+1}^{* \prime}+\frac{1}{2} w_{m+1}^{* / 2}\right) d x^{*}\right],
\end{aligned}
$$

where $(\cdot)$ denotes the derivative with respect to time $\left(t^{*}\right)$, and ()$^{\prime}$ denotes the derivative with respect to the spatial variable $\left(x^{*}\right)$. In Eq. (1) the rotary inertia and shear effect are not included, and cross-sectional area does not change during motion. $x_{m+1}^{*}$ denotes the distance between any support and the origin. $m=$ $0,1,2, \ldots, n$, where $n$ is the number of supports. The first two integrals inside the summation sign are kinetic energies between any successive supports (e.g., 
1st-2nd, 2nd-3rd, 3rd-4th, and so on). The terms in the second summation sign are elastic potential energies due to elongation, bending, and tensile force $(P)$ between any successive supports, respectively. $x_{0}=0$, and $x_{n+1}=L$ is the total length, $x_{p}$ is the location of multiple supports. The material properties in the equation are defined as follows: $\rho A$ is the mass per unit length, $E A$ is the longitudinal rigidity, and $E I$ is the flexural rigidity. After applying Hamilton's principle to Eq. (1), the equations of motion between any successive supports can be obtained as follows:

$$
\begin{aligned}
& \left(\ddot{w}_{m+1}^{*}+2 \dot{w}_{m+1}^{* \prime} v^{*}+w_{m+1}^{* \prime} \dot{v}^{*}+w_{m+1}^{* \prime \prime} v^{* 2}\right) \\
& +\frac{E I}{\rho A} w_{m+1}^{* i v}-\frac{P}{\rho A} w_{m+1}^{* \prime \prime} \\
& -\frac{E}{\rho}\left(u_{m+1}^{* \prime \prime} w_{m+1}^{* \prime}+u_{m+1}^{* \prime} w_{m+1}^{* \prime \prime}\right. \\
& \left.\quad+\frac{3}{2} w_{m+1}^{* \prime 2} w_{m+1}^{* \prime \prime}\right)=0, \\
& \left(\ddot{u}_{m+1}^{*}+2 \dot{u}_{m+1}^{* \prime} v^{*}+u_{m+1}^{* \prime} \dot{v}^{*}+\dot{v}^{*}+u_{m+1}^{* \prime \prime} v^{* 2}\right) \\
& \quad-\frac{E}{\rho}\left(u_{m+1}^{* \prime}+\frac{1}{2} w_{m+1}^{* \prime 2}\right)^{\prime}=0 .
\end{aligned}
$$

Using the following parameters, one can make the equations nondimensional:

$w_{m+1}=\frac{w_{m+1}^{*}}{L}, \quad u_{m+1}=\frac{u_{m+1}^{*}}{L}, \quad \eta=\frac{x_{m+1}^{*}}{L}$,

$t=t^{*} \sqrt{\frac{P}{\rho A L^{2}}}$,

$v=\frac{v^{*}}{\sqrt{P / \rho A}}, \quad v_{b}^{2}=\frac{E A}{P}, \quad \bar{v}_{f}^{2}=\frac{E I}{P L^{2}}$,

where $v_{b}$ represents the longitudinal rigidity, and $\bar{v}_{f}$ is the flexural rigidity. The axial velocity is made nondimensional by dividing with critical velocity. The explanation for $v_{b}^{2} \gg 1$ is given in reference [4]. After performing necessary mathematical operations and including damping, nondimensional integro-differential equations of motion and boundary conditions for the general case are obtained as follows:

$$
\begin{gathered}
\left(\ddot{w}_{m+1}+2 \dot{w}_{m+1}^{\prime} v+w_{m+1}^{\prime} \dot{v}\right)+\left(v^{2}-1\right) w_{m+1}^{\prime \prime} \\
+\bar{v}_{f}^{2} w_{m+1}^{i v}+\bar{\mu}\left(\dot{w}_{m+1}+v w_{m+1}^{\prime}\right)
\end{gathered}
$$

$$
=\frac{1}{2} v_{b}^{2}\left(\sum_{r=0}^{n} \int_{\eta_{r}}^{\eta_{r+1}} w_{r+1}^{\prime 2} d x\right) w_{m+1}^{\prime \prime},
$$

$m=0,1,2 \ldots n, \quad \eta_{0}=0, \quad \eta_{n+1}=1$,

$w_{1}(0, t)=0, \quad w_{n+1}(1, t)=0, \quad w_{1}^{\prime \prime}(0, t)=0$,

$w_{n+1}^{\prime \prime}(1, t)=0$,

$w_{p}\left(\eta_{p}, t\right)=0, \quad w_{p+1}\left(\eta_{p}, t\right)=0$,

$w_{p}^{\prime}\left(\eta_{p}, t\right)=w_{p+1}^{\prime}\left(\eta_{p}, t\right), \quad w_{p}^{\prime \prime}\left(\eta_{p}, t\right)=w_{p+1}^{\prime \prime}\left(\eta_{p}, t\right)$

$(p=1,2,3, \ldots, n)$.

The right-hand side of the equation above represents the stretching of the neutral axis. $\ddot{w}_{m+1}$ is the local acceleration, $2 \dot{w}_{m+1}^{\prime} v$ is the Coriolis acceleration, $v_{b}^{2} w_{m+1}^{\prime \prime}$ is the centripetal acceleration, and $\eta_{p}$ are the locations of intermediate supports. The transport velocity with constant mean and arbitrary fluctuation frequency can be written as follows:

$v=v_{0}+\varepsilon v_{1} \sin \Omega t$,

where $\varepsilon$ denotes a small variation. The displacement in Eq. (5) can be assumed as $w_{m+1}=\sqrt{\varepsilon} y_{m+1}$ to guarantee that the longitudinal rigidity depending on nonlinear effects appears in higher orders of expansion. Using Eqs. (5) and (6), the equation of motion and boundary conditions become

$$
\begin{aligned}
& \ddot{y}_{m+1}+2 \dot{y}_{m+1}^{\prime} v_{0}+2 \varepsilon \dot{y}_{m+1}^{\prime} v_{1} \sin \Omega t \\
& \quad+\varepsilon y_{m+1}^{\prime} v_{1} \Omega \cos \Omega t+\bar{v}_{f}^{2} y_{m+1}^{i v} \\
& \quad+\varepsilon \bar{\mu}\left(\dot{y}_{m+1}+v_{0} y_{m+1}^{\prime}\right)+\left(v_{0}^{2}\right. \\
& \left.\quad+\varepsilon^{2} v_{1}^{2} \sin ^{2} \Omega t+2 \varepsilon v_{0} v_{1} \sin \Omega t-1\right) y_{m+1}^{\prime \prime} \\
& =\frac{1}{2} v_{b}^{2} \varepsilon y_{m+1}^{\prime \prime}\left(\sum_{r=0}^{n} \int_{\eta_{r}}^{\eta_{r+1}} y_{r+1}^{\prime 2} d x\right) y_{m+1}^{\prime \prime}, \\
& y_{1}(0, t)=0, \quad y_{1}^{\prime \prime}(0, t)=0, \quad y_{n+1}(1, t)=0, \\
& y_{p}\left(\eta_{p}, t\right)=0, \quad y_{p+1}\left(\eta_{p}, t\right)=0, \\
& y_{p}^{\prime}\left(\eta_{p+1}, t\right)=y_{p+1}^{\prime}\left(\eta_{p, t}\right)=0\left(\eta_{p}, t\right), \\
& y_{p}^{\prime \prime}\left(\eta_{p}, t\right)=y_{p+1}^{\prime \prime}\left(\eta_{p}, t\right) .
\end{aligned}
$$

One can make an arrangement for the orders of flexural rigidity and viscous damping as $\bar{v}_{f}^{2}=v_{f}^{2}$ and $\bar{\mu}=\varepsilon \mu$. These equations will be solved analytically in the next section. 


\section{Perturbation analysis}

For searching the approximate solutions of Eq. (7), the method of multiple scales will be used. The displacement functions for sections between any successive two supports can be expanded as shown below:

$$
\begin{aligned}
y_{m+1}(x, t ; \varepsilon)= & \left.y_{(m+1) 1}\left(x, T_{0}, T_{1}\right)\right) \\
& +\varepsilon y_{(m+1) 2}\left(x, T_{0}, T_{1}+\cdots,\right.
\end{aligned}
$$

where $T_{0}=t$ and $T_{1}=\varepsilon t$ are the slow and fast time scales, respectively. The first and second time derivatives used in Eq. (7) are defined as follows:

$\frac{d}{d t}=D_{0}+\varepsilon D_{1}+\cdots$,

$\frac{d^{2}}{d t^{2}}=D_{0}^{2}+2 \varepsilon D_{0} D_{1}+\cdots$,

where $D_{i}=\partial / \partial T_{i}$. Substituting Eqs. (8), (9) into Eq. (7), one obtains equations at different orders of perturbation expansion:

$O(1)$ :

$D_{0}^{2} y_{(m+1) 1}+2 v_{0} D_{0} y_{(m+1) 1}^{\prime}+\left(v_{0}^{2}-1\right) y_{(m+1) 1}^{\prime \prime}$

$+v_{f}^{2} y_{(m+1) 1}^{i v}=0$,

$y_{1}(0, t)=0, \quad y_{1}^{\prime \prime}(0, t)=0, \quad y_{n+1}(1, t)=0$,

$y_{n+1}^{\prime \prime}(1, t)=0$,

$y_{p}\left(\eta_{p}, t\right)=0, \quad y_{p+1}\left(\eta_{p}, t\right)=0$,

$y_{p}^{\prime}\left(\eta_{p+1}, t\right)=y_{p+1}^{\prime}\left(\eta_{p}, t\right), \quad y_{p}^{\prime \prime}\left(\eta_{p}, t\right)=y_{p+1}^{\prime \prime}\left(\eta_{p}, t\right)$.

$O(\varepsilon):$

$$
\begin{aligned}
D_{0}^{2} y_{(m+1) 2}+2 v_{0} D_{0} y_{(m+1) 2}^{\prime}+v_{f}^{2} y_{(m+1) 2}^{i v} & \\
& +\left(v_{0}^{2}-1\right) y_{(m+1) 2}^{\prime \prime} \\
= & -2 D_{0} D_{1} y_{(m+1) 1}-2 v_{0} D_{1} y_{(m+1) 1}^{\prime} \\
& -2 v_{1} \sin \Omega t D_{0} y_{(m+1) 1}^{\prime}-2 y_{(m+1) 1}^{\prime \prime} v_{0} v_{1} \sin \Omega t \\
& -y_{(m+1) 1}^{\prime} v_{1} \Omega \cos \Omega t-\mu D_{0} y_{(m+1) 1} \\
& -\mu v_{0} y_{(m+1) 1}^{\prime} \\
& +\frac{1}{2} v_{b}^{2}\left(\sum_{r=0}^{n} \int_{\eta_{r}}^{\eta_{r+1}} y_{(m+1) 1}^{\prime 2} d x\right) y_{(m+1) 1}^{\prime \prime},
\end{aligned}
$$

$y_{1}(0, t)=0, \quad y_{1}^{\prime \prime}(0, t)=0$, $y_{n+1}(1, t)=0, \quad y_{n+1}^{\prime \prime}(1, t)=0$,

$y_{p}\left(\eta_{p}, t\right)=0, \quad y_{p+1}\left(\eta_{p}, t\right)=0$,

$y_{p}^{\prime}\left(\eta_{p+1}, t\right)=y_{p+1}^{\prime}\left(\eta_{p}, t\right)$,

$y_{p}^{\prime \prime}\left(\eta_{p}, t\right)=y_{p+1}^{\prime \prime}\left(\eta_{p}, t\right)$.

The solution of Eq. (10) will give velocity-dependent natural frequencies and mode shapes between adjacent two supports. It can be assumed as follows:

$$
\begin{aligned}
y_{(m+1) 1}\left(x, T_{0}, T_{1} ; \varepsilon\right)= & A\left(T_{1}\right) e^{i \omega T_{0}} Y_{(m+1)}(x) \\
& +\bar{A}\left(T_{1}\right) e^{-i \omega T_{0}} \bar{Y}_{(m+1)}(x) .
\end{aligned}
$$

Inserting it into the equations gives

$$
\begin{aligned}
& v_{f}^{2} Y_{m+1}^{i v}+\left(v_{0}^{2}-1\right) Y_{m+1}^{\prime \prime}+2 i v_{0} \omega Y_{m+1}^{\prime}-\omega^{2} Y_{m+1} \\
& \quad=0, \\
& Y_{1}(0)=0, \quad Y_{n+1}(1)=0, \quad Y_{1}^{\prime \prime}(0)=0, \\
& Y_{n+1}^{\prime \prime}(1)=0, \\
& Y_{p}\left(\eta_{p}\right)=0, \quad Y_{p+1}\left(\eta_{p}\right)=0, \quad Y_{p}^{\prime}\left(\eta_{p}\right)=Y_{p+1}^{\prime}\left(\eta_{p}\right), \\
& Y_{p}^{\prime \prime}\left(\eta_{p}\right)=Y_{p+1}^{\prime \prime}\left(\eta_{p}\right) .
\end{aligned}
$$

The following functions can be proposed for the solutions of equations above:

$$
\begin{aligned}
Y_{m+1}(x)= & c_{4 m+1}\left(e^{i \beta_{4 m+1} x}+c_{4 m+2} e^{i \beta_{4 m+2} x}\right. \\
& \left.+c_{4 m+3} e^{i \beta_{4 m+3} x}+c_{4 m+4} e^{i \beta_{4 m+4} x}\right) .
\end{aligned}
$$

Then we obtain the dispersion relation

$$
\begin{aligned}
& v_{f}^{2} \beta_{4 m+1}^{4}+\left(1-v_{0}^{2}\right) \beta_{4 m+1}^{2}-2 \omega v_{0} \beta_{4 m+1}-\omega^{2}=0 \\
& \quad(m=0,1,2, \ldots, n) .
\end{aligned}
$$

The support condition is obtained by applying the boundary conditions similar to references $[4,14,15]$ (for two supports only) for multiple support case as given below:

$$
\begin{aligned}
& {\left[v_{f}^{2} \beta_{4 m+1}^{4}+\left(1-v_{0}^{2}\right) \beta_{4 m+1}^{2}-2 \omega v_{0} \beta_{4 m+1}\right.} \\
& \left.\quad-\omega^{2}\right] e^{i \beta_{4 m+1} x} \\
& \quad+\left[v_{f}^{2} \beta_{4 m+2}^{4}+\left(1-v_{0}^{2}\right) \beta_{4 m+2}^{2}-2 \omega v_{0} \beta_{4 m+2}\right. \\
& \left.\quad-\omega^{2}\right] c_{4 m+2} e^{i \beta_{4 m+2} x} \\
& \quad+\left[v_{f}^{2} \beta_{4 m+3}^{4}+\left(1-v_{0}^{2}\right) \beta_{4 m+3}^{2}-2 \omega v_{0} \beta_{4 m+3}\right.
\end{aligned}
$$




$$
\begin{aligned}
& \left.-\omega^{2}\right] c_{4 m+3} e^{i \beta_{4 m+3} x} \\
& +\left[v_{f}^{2} \beta_{4 m+4}^{4}+\left(1-v_{0}^{2}\right) \beta_{4 m+4}^{2}-2 \omega v_{0} \beta_{4 m+4}\right. \\
& \left.-\omega^{2}\right] c_{4 m+4} e^{i \beta_{4 m+4} x}=0 .
\end{aligned}
$$

The coefficients in function (14) can be obtained from the boundary conditions (13) in terms of one of the coefficients by equating the determinant of the following matrix to zero. Numerical examples for linear frequencies considering different cases will be presented later. Support condition matrices for 3- and 4-support cases and for a general form of the multiple support case are given in the Appendix.

Order $\varepsilon(11)$ represents the nonlinear behavior. The following functions can be proposed for the solution:

$$
\begin{aligned}
y_{(m+1) 2}\left(x, T_{0}, T_{1} ; \varepsilon\right)= & \phi_{m+1}\left(x, T_{1}\right) e^{i \omega T_{0}} \\
& +W_{m+1}\left(x, T_{0}, T_{1}\right)+c c,
\end{aligned}
$$

where the first term $\left(\phi_{m+1}\right)$ is related with the secular terms, the second one $\left(W_{m+1}\right)$ is related with the nonsecular terms (NST), and $c c$ stands for the complex conjugate of the preceding terms. After using these substitutions, one gets

$$
\begin{aligned}
\left(v_{f}^{2} \phi_{m+1}^{i v}+\left(v_{0}^{2}-1\right) \phi_{m+1}^{\prime \prime}+2 i v_{0} \omega \phi_{m+1}^{\prime}\right. \\
\left.\quad-\omega^{2} \phi_{m+1}\right) e^{i \omega T_{0}} \\
=-2\left(i \omega Y_{m+1}+v_{0} Y_{m+1}^{\prime}\right) D_{1} A e^{i \omega T_{0}} \\
+v_{1}\left(-\omega Y_{m+1}^{\prime}-\frac{\Omega}{2} Y_{m+1}^{\prime}+i v_{0} Y_{m+1}^{\prime \prime}\right) \\
\quad \times A e^{i(\Omega+\omega) T_{0}}+v_{1}\left(\omega \bar{Y}_{m+1}^{\prime}-\frac{\Omega}{2} \bar{Y}_{m+1}^{\prime}\right. \\
\left.+i v_{0} \bar{Y}_{m+1}^{\prime \prime}\right) \bar{A} e^{i(\Omega-\omega) T_{0}}
\end{aligned}
$$

$$
\begin{aligned}
& -\mu\left(i \omega Y_{m+1}+v_{0} Y_{m+1}^{\prime}\right) A e^{i \omega T_{0}} \\
& +\frac{1}{2} v_{b}^{2}\left[\bar{Y}_{m+1}^{\prime \prime}\left(\sum_{r=0}^{n} \int_{\eta_{r}}^{\eta_{r+1}} Y_{m+1}^{\prime 2} d x\right)\right. \\
& \left.+2 Y_{m+1}^{\prime \prime}\left(\sum_{r=0}^{n} \int_{\eta_{r}}^{\eta_{r+1}} Y_{m+1}^{\prime} \bar{Y}_{m+1}^{\prime} d x\right)\right] \\
& \times A^{2} \bar{A} e^{i \omega T_{0}}+c c+N S T, \\
& m=0,1,2, \ldots, n, \quad \eta_{0}=0, \quad \eta_{n+1}=1 .
\end{aligned}
$$

Since the left-hand sides of Eqs. (10) and (11) are the same, and Eq. (10) has a nontrivial solution, a solvability condition should be obtained for Eq. (11) to have a solution. A function is chosen depending on whether the problem is adjoint or self-adjoint. For this case, the function is adjoint. The solvability condition can be obtained by following reference [32]. These solutions will be discussed for different velocity fluctuation frequencies $\Omega$.

\subsection{Principal parametric resonances}

When the velocity fluctuation frequency is close to two times any natural frequency, the principal parametric resonance will occur. This case can be represented by

$\Omega=2 \omega+\varepsilon \sigma$,

where $\sigma$ is a detuning parameter. The solvability condition can be obtained as follows:

$D_{1} A+k_{0} \bar{A} e^{i \sigma T_{1}}+\frac{\mu}{2} A-k_{3} A^{2} \bar{A}=0$,

where

$$
\begin{aligned}
& k_{0}=v_{1} \frac{\left[\left(\frac{\Omega}{2}-\omega\right)\left(\sum_{r=0}^{n} \int_{\eta_{r}}^{\eta_{r+1}} \bar{Y}_{m+1}^{\prime} \bar{Y}_{m+1} d x\right)-i v_{0}\left(\sum_{r=0}^{n} \int_{\eta_{r}}^{\eta_{r+1}} \bar{Y}_{m+1}^{\prime \prime} \bar{Y}_{m+1} d x\right)\right]}{2\left[i \omega\left(\sum_{r=0}^{n} \int_{\eta_{r}}^{\eta_{r+1}} Y_{m+1} \bar{Y}_{m+1} d x\right)+v_{0}\left(\sum_{r=0}^{n} \int_{\eta_{r}}^{\eta_{r+1}} Y_{m+1}^{\prime} \bar{Y}_{m+1} d x\right)\right]}, \\
& k_{3}=\frac{1}{2} v_{b}^{2} \frac{\left[\sum_{r=0}^{n}\left(\int_{\eta_{r}}^{\eta_{r+1}} \bar{Y}_{m+1}^{\prime \prime} \bar{Y}_{m+1} d x\right)\left(\int_{\eta_{r}}^{\eta_{r+1}} Y_{m+1}^{\prime 2} d x\right)+2 \sum_{r=0}^{n}\left(\int_{\eta_{r}}^{\eta_{r+1}} Y_{m+1}^{\prime \prime} \bar{Y}_{m+1} d x\right)\left(\int_{\eta_{r}}^{\eta_{r+1}} Y_{m+1}^{\prime} \bar{Y}_{m+1}^{\prime} d x\right)\right]}{2\left[i \omega\left(\sum_{r=0}^{n} \int_{\eta_{r}}^{\eta_{r+1}} Y_{m+1} \bar{Y}_{m+1} d x\right)+v_{0}\left(\sum_{r=0}^{n} \int_{\eta_{r}}^{\eta_{r+1}} Y_{m+1}^{\prime} \bar{Y}_{m+1} d x\right)\right]} .
\end{aligned}
$$

The amplitude in Eq. (20) can be written in polar form as

$A=\frac{1}{2} a e^{i \theta}$.
Amplitude and phase modulation equations are obtained by separating Eq. (21) into real and imaginary 
parts as follows:

$D_{1} a=a\left(k_{0_{\mathrm{I}}} \sin \gamma-k_{0_{R}} \cos \gamma+\frac{1}{2} \mu\right)$,

$a D_{1} \gamma=a \sigma+2 a\left(k_{0_{\mathrm{I}}} \cos \gamma+k_{0_{R}} \sin \gamma\right)-\frac{1}{2} k_{3_{\mathrm{I}}} a^{3}$,

where $k_{0}=k_{0_{R}}+i k_{0_{\mathrm{I}}}, k_{3}=i k_{3_{\mathrm{I}}}$, and the real part of $k_{3}$ is very small compared to the real part [14], and

$\gamma=\sigma T_{1}-2 \theta$.

The transformation can be assumed for the phase to seek the solution in steady-state region $D_{1} a=0$, $D_{1} \gamma=0$ :

$$
\begin{aligned}
F_{1}(a, \gamma)= & \left(k_{0_{\mathrm{I}}} \sin \gamma-k_{0_{R}} \cos \gamma+\frac{1}{2} \mu\right), \\
F_{2}(a, \gamma)= & \sigma+2\left(k_{0_{\mathrm{I}}} \cos \gamma+k_{0_{R}} \sin \gamma\right) \\
& -\frac{1}{2} k_{3_{\mathrm{I}}} a^{2} .
\end{aligned}
$$

The solution of phase modulation equations with zero amplitude is the trivial solution, and the other case is the nontrivial solution. The relation between the velocity fluctuation frequency and amplitude of the nontrivial solution can be obtained as follows:

$\sigma_{1,2}=\frac{1}{2} a^{2} k_{3_{\mathrm{I}}} \mp 2 \sqrt{k_{0_{\mathrm{I}}}^{2}+k_{0_{R}}^{2}-\frac{1}{4} \mu^{2}}$.

The Jacobian matrix can be constructed to investigate the stability conditions of the nontrivial solution:

$$
\left[\begin{array}{ll}
\frac{\partial F_{1}}{\partial a} & \frac{\partial F_{1}}{\partial \gamma} \\
\frac{\partial F_{2}}{\partial a} & \frac{\partial F_{2}}{\partial \gamma}
\end{array}\right]_{\substack{a=a_{0} \\
\gamma=\gamma_{0}}} .
$$

The eigenvalues are obtained from the equation

$$
\left[\begin{array}{cc}
-\lambda & a\left(\frac{1}{4} k_{3_{\mathrm{I}}} a^{2}-\frac{\sigma}{2}\right) \\
-k_{3_{\mathrm{I}}} a & -\mu-\lambda
\end{array}\right]_{\substack{a=a_{0} \\
\gamma=\gamma_{0}}}
$$

as follows:

$$
\lambda_{1,2}=\frac{1}{2}\left(-\mu \mp \sqrt{\mu+2 a^{2} \sigma k_{3_{\mathrm{I}}}-a^{4} k_{3_{\mathrm{I}}}^{2}}\right) .
$$

The complex amplitudes in the polar form

$$
A=\frac{1}{2}(p+i q) a e^{i \frac{\sigma}{2} T_{1}}
$$

are rewritten for the stability analysis of the trivial solution. Then phase modulation equations are obtained as follows:

$$
\begin{aligned}
D_{1} p= & -\left(k_{0_{R}}-\frac{1}{2} \mu\right) p+\left(\frac{\sigma}{2}-k_{0_{\mathrm{I}}}\right) q \\
& -\frac{1}{4} k_{3_{\mathrm{I}}} q\left(p^{2}-q^{2}\right), \\
D_{1} q= & \left(k_{0_{R}}-\frac{1}{2} \mu\right) q+\left(\frac{\sigma}{2}+k_{0_{\mathrm{I}}}\right) p \\
& +\frac{1}{4} k_{3_{\mathrm{I}}} p\left(p^{2}+q^{2}\right) .
\end{aligned}
$$

The eigenvalues are

$\lambda_{1,2}=\frac{1}{2}\left(-\mu \mp \sqrt{4\left(k_{0_{\mathrm{I}}}^{2}+k_{0_{R}}^{2}\right)-\sigma^{2}}\right)$,

and the stability boundaries for the trivial solution are

$$
2 \sqrt{k_{0_{\mathrm{I}}}^{2}+k_{0_{R}}^{2}-\frac{1}{4} \mu^{2}} \leq \sigma \leq-2 \sqrt{k_{0_{\mathrm{I}}}^{2}+k_{0_{R}}^{2}-\frac{1}{4} \mu^{2}} .
$$

There will be no nontrivial solutions in the regions at which the trivial solutions are stable [33]. There exist nontrivial solutions in the regions at which the trivial solutions are unstable. In the latter case, amplitudes of vibration increase.

\subsection{1 $\Omega$ is away from 0 and $2 \omega$}

This is the case for the velocity fluctuation frequency away from 0 and $2 \omega$. The solvability condition is

$D_{1} A+\frac{\mu}{2} A-k_{3} A^{2} \bar{A}=0$,

and phase modulation equations are

$D_{1} a+\frac{\mu}{2} a=0$,

$a D_{1} \theta-\frac{1}{4} k_{3_{I}} a^{3}=0$.

For undamped free vibrations, $\mu=0$, the amplitude is constant $\left(a=a_{0}\right)$, and the phase is

$\theta=\frac{1}{4} k_{3_{\mathrm{I}}} a^{2} T_{1}+\beta_{0}$. 
The nonlinear natural frequency is obtained as follows:

$\omega_{n l}=\omega+\varepsilon\left(\frac{1}{4} k_{3_{\mathrm{I}}} a^{2}\right)$.

\subsection{2 $\Omega$ is close to 0}

The velocity fluctuation frequency is

$\Omega=\varepsilon \sigma$.
The solvability condition is

$$
D_{1} A+\frac{\mu}{2} A+\left(k_{1} \cos \sigma T_{1}+k_{2} \sin \sigma T_{1}\right) A-k_{3} A^{2} \bar{A}=0,
$$

where

$$
\begin{aligned}
& k_{1}=\frac{v_{1} \Omega\left(\sum_{r=0}^{n} \int_{\eta_{r}}^{\eta_{r+1}} Y_{m+1}^{\prime} \bar{Y}_{m+1} d x\right)}{2\left[i \omega\left(\sum_{r=0}^{n} \int_{\eta_{r}}^{\eta_{r+1}} Y_{m+1} \bar{Y}_{m+1} d x\right)+v_{0}\left(\sum_{r=0}^{n} \int_{\eta_{r}}^{\eta_{r+1}} Y_{m+1}^{\prime} \bar{Y}_{m+1} d x\right)\right]}, \\
& k_{2}=\frac{i v_{1} \omega\left(\int_{0}^{\eta} Y_{1}^{\prime} \bar{Y}_{1} d x+\int_{\eta}^{1} Y_{2}^{\prime} \bar{Y}_{2} d x\right)}{\left[i \omega\left(\sum_{r=0}^{n} \int_{\eta_{r}}^{\eta_{r+1}} Y_{m+1} \bar{Y}_{m+1} d x\right)+v_{0}\left(\sum_{r=0}^{n} \int_{\eta_{r}}^{\eta_{r+1}} Y_{m+1}^{\prime} \bar{Y}_{m+1} d x\right)\right]} .
\end{aligned}
$$

The amplitude for this case is

$a=a_{o} e^{-\mu T_{1}+\frac{\left(k_{1} \cos \sigma T_{1}-k_{2} \sin \sigma T_{1}\right)}{\sigma}}$.

Since $\left|\sin \sigma T_{1}\right| \leq 1$ and $\left|\cos \sigma T_{1}\right| \leq 1$, the complex amplitudes are bounded in time, and thus there is no instability for this case.

\subsection{Combination resonances}

In this section, we assume that there are two dominant modes. Two cases are significant. The velocity variation frequency may either be nearly equal to the sum of any two modes or to the difference of any two modes. One can assume the following function for the solution of Eqs. (10) for combination resonances in which the $a$ th and $b$ th modes are effective:

$$
\begin{aligned}
y_{(m+1) 1}\left(x, T_{0}, T_{1} ; \varepsilon\right)= & A_{a}\left(T_{1}\right) e^{i \omega_{a} T_{0}} Y_{(m+1), a}(x) \\
& +A_{b}\left(T_{1}\right) e^{i \omega_{b} T_{0}} Y_{(m+1), b}(x) \\
& +c c .
\end{aligned}
$$

Shape functions for the two modes can be proposed as follows:

$$
\begin{aligned}
Y_{(m+1), p}(x)= & c_{4 m+1, p} e^{i \beta_{4 m+1} x}+c_{4 m+2, p} e^{i \beta_{4 m+2} x} \\
& +c_{4 m+3, p} e^{i \beta_{4 m+3} x}+c_{4 m+4, p} e^{i \beta_{4 m+4} x},
\end{aligned}
$$$$
p=a, b .
$$

Inserting the function above into the first order of expansion gives

$$
\begin{aligned}
& v_{f}^{2} Y_{(m+1), p}^{i v}+\left(v_{0}^{2}-1\right) Y_{(m+1), p}^{\prime \prime}+2 i v_{0} \omega_{p} Y_{(m+1), p}^{\prime} \\
& \quad-\omega_{p}^{2} Y_{(m+1), p}=0 .
\end{aligned}
$$

The displacement function for the second order of expansion can be defined as follows:

$$
\begin{aligned}
& y_{(m+1) 2}\left(x, T_{0}, T_{1} ; \varepsilon\right) \\
& =\phi_{(m+1), a}\left(x, T_{1}\right) e^{i \omega_{a} T_{0}}+\phi_{(m+1), b} e^{i \omega_{b} T_{0}} \\
& \quad+W_{(m+1)}\left(x, T_{0}, T_{1}\right)+c c .
\end{aligned}
$$

The first two terms are related with secular terms in the $a$ th and $b$ th modes, the third one is related with nonsecular terms. Inserting the functions defined above into the second order of expansion, we get

$$
\begin{aligned}
& \left(v_{f}^{2} \phi_{(m+1), a}^{i v}+\left(v_{0}^{2}-1\right) \phi_{(m+1), a}^{\prime \prime}+2 i v_{0} \omega_{a} \phi_{(m+1), a}^{\prime}-\omega_{a}^{2} \phi_{(m+1), a}\right) e^{i \omega_{a} T_{0}} \\
& \quad+\left(v_{f}^{2} \phi_{(m+1), b}^{i v}+\left(v_{0}^{2}-1\right) \phi_{(m+1), b}^{\prime \prime}+2 i v_{0} \omega_{a} \phi_{(m+1), b}^{\prime}-\omega_{a}^{2} \phi_{(m+1), b}\right) e^{i \omega_{b} T_{0}}
\end{aligned}
$$




$$
\begin{aligned}
& =-\left(2 D_{1} A_{a}+\mu A_{a}\right)\left(i \omega_{a} Y_{(m+1), a}+v_{0} Y_{(m+1) a}^{\prime}\right) e^{i \omega_{a} T_{0}}-\left(2 D_{1} A_{b}+\mu A_{b}\right)\left(i \omega_{b} Y_{(m+1), b}+v_{0} Y_{(m+1), b}^{\prime}\right) e^{i \omega_{b} T_{0}} \\
& +A_{a} v_{1}\left(-\omega_{a} Y_{(m+1), a}^{\prime}-\frac{\Omega}{2} Y_{(m+1), a}^{\prime}+i v_{0} Y_{(m+1), a}^{\prime \prime}\right) e^{i\left(\Omega+\omega_{a}\right) T_{0}} \\
& +A_{b} v_{1}\left(-\omega_{b} Y_{(m+1), b}^{\prime}-\frac{\Omega}{2} Y_{(m+1), b}^{\prime}+i v_{0} Y_{(m+1), b}^{\prime \prime}\right) e^{i\left(\Omega+\omega_{b}\right) T_{0}} \\
& +\bar{A}_{a} v_{1}\left(\omega_{a} \bar{Y}_{(m+1), a}^{\prime}-\frac{\Omega}{2} \bar{Y}_{(m+1), a}^{\prime}+i v_{0} \bar{Y}_{(m+1), a}^{\prime \prime}\right) e^{i\left(\Omega-\omega_{a}\right) T_{0}} \\
& +\bar{A}_{b} v_{1}\left(\omega_{b} \bar{Y}_{(m+1), b}^{\prime}-\frac{\Omega}{2} \bar{Y}_{(m+1), b}^{\prime}+i v_{0} \bar{Y}_{(m+1), b}^{\prime \prime}\right) e^{i\left(\Omega-\omega_{b}\right) T_{0}} \\
& +\frac{1}{2} v_{b}^{2}\left[\left[A_{a}^{3}\left(\sum_{r=0}^{n} \int_{\eta_{r}}^{\eta_{r+1}} Y_{(m+1), a}^{\prime 2} d x\right) e^{3 i \omega_{a} T_{0}}+A_{a} \bar{A}_{a}^{2}\left(\sum_{r=0}^{n} \int_{\eta_{r}}^{\eta_{r+1}} \bar{Y}_{(m+1), a}^{\prime 2} d x\right) e^{-i \omega_{a} T_{0}}\right.\right. \\
& +2 A_{a} \bar{A}_{a} \bar{A}_{b}\left(\sum_{r=0}^{n} \int_{\eta_{r}}^{\eta_{r+1}} \bar{Y}_{(m+1), a}^{\prime} \bar{Y}_{(m+1), b}^{\prime} d x\right) e^{-i \omega_{b} T_{0}}+A_{a} \bar{A}_{b}^{2}\left(\sum_{r=0}^{n} \int_{\eta_{r}}^{\eta_{r+1}} \bar{Y}_{(m+1), b}^{\prime 2} d x\right) e^{i\left(\omega_{a}-2 \omega_{b}\right) T_{0}} \\
& +2 A_{a}^{2} \bar{A}_{a}\left(\sum_{r=0}^{n} \int_{\eta_{r}}^{\eta_{r+1}} Y_{(m+1), a}^{\prime} \bar{Y}_{(m+1), a}^{\prime} d x\right) e^{i \omega_{a} T_{0}}+2 A_{a}^{2} \bar{A}_{b}\left(\sum_{r=0}^{n} \int_{\eta_{r}}^{\eta_{r+1}} Y_{(m+1), a}^{\prime} \bar{Y}_{(m+1), b}^{\prime} d x\right) e^{i\left(2 \omega_{a}-\omega_{b}\right) T_{0}} \\
& +2 A_{a} \bar{A}_{a} A_{b}\left(\sum_{r=0}^{n} \int_{\eta_{r}}^{\eta_{r+1}} \bar{Y}_{(m+1), a}^{\prime} Y_{(m+1), b}^{\prime} d x\right) e^{i \omega_{b} T_{0}}+2 A_{a} A_{b} \bar{A}_{b}\left(\sum_{r=0}^{n} \int_{\eta_{r}}^{\eta_{r+1}} Y_{(m+1), b}^{\prime} \bar{Y}_{(m+1), b}^{\prime} d x\right) e^{i \omega_{a} T_{0}} \\
& +2 A_{a}^{2} A_{b}\left(\sum_{r=0}^{n} \int_{\eta_{r}}^{\eta_{r+1}} Y_{(m+1), a}^{\prime} Y_{(m+1), b}^{\prime} d x\right) e^{i\left(2 \omega_{a}+\omega_{b}\right) T_{0}} \\
& \left.+A_{a} A_{b}^{2}\left(\sum_{r=0}^{n} \int_{\eta_{r}}^{\eta_{r+1}} \bar{Y}_{(m+1), b}^{\prime 2} d x\right) e^{i\left(\omega_{a}+2 \omega_{b}\right) T_{0}}\right] Y_{(m+1), a}^{\prime \prime} \\
& +\left[A_{b}^{3}\left(\sum_{r=0}^{n} \int_{\eta_{r}}^{\eta_{r+1}} Y_{(m+1), b}^{\prime 2} d x\right) e^{3 i \omega_{b} T_{0}}+\bar{A}_{a}^{2} A_{b}\left(\sum_{r=0}^{n} \int_{\eta_{r}}^{\eta_{r+1}} \bar{Y}_{(m+1), a}^{2} d x\right) e^{i\left(-2 \omega_{a}+\omega_{b}\right) T_{0}}\right. \\
& +2 \bar{A}_{a} A_{b} \bar{A}_{b}\left(\sum_{r=0}^{n} \int_{\eta_{r}}^{\eta_{r+1}} \bar{Y}_{(m+1), a}^{\prime} \bar{Y}_{(m+1), b}^{\prime} d x\right) e^{-i \omega_{a} T_{0}}+A_{b} \bar{A}_{b}^{2}\left(\sum_{r=0}^{n} \int_{\eta_{r}}^{\eta_{r+1}} \bar{Y}_{(m+1), b}^{\prime 2} d x\right) e^{-i \omega_{b} T_{0}} \\
& +2 A_{a} \bar{A}_{a} A_{b}\left(\sum_{r=0}^{n} \int_{\eta_{r}}^{\eta_{r+1}} Y_{(m+1), a}^{\prime} \bar{Y}_{(m+1), a}^{\prime} d x\right) e^{i \omega_{b} T_{0}}+2 A_{a} A_{b} \bar{A}_{b}\left(\sum_{r=0}^{n} \int_{\eta_{r}}^{\eta_{r+1}} Y_{(m+1), a}^{\prime} \bar{Y}_{(m+1), b}^{\prime} d x\right) e^{i \omega_{a} T_{0}} \\
& +2 A_{a}^{2} A_{b}\left(\sum_{r=0}^{n} \int_{\eta_{r}}^{\eta_{r+1}} Y_{(m+1), a}^{\prime 2} d x\right) e^{i\left(2 \omega_{a}+\omega_{b}\right) T_{0}}+2 \bar{A}_{a} A_{b}^{2}\left(\sum_{r=0}^{n} \int_{\eta_{r}}^{\eta_{r+1}} \bar{Y}_{(m+1), a}^{\prime} Y_{(m+1), b}^{\prime} d x\right) e^{i\left(-\omega_{a}+2 \omega_{b}\right) T_{0}} \\
& +2 A_{b}^{2} \bar{A}_{b}\left(\sum_{r=0}^{n} \int_{\eta_{r}}^{\eta_{r+1}} Y_{(m+1), b}^{\prime} \bar{Y}_{(m+1), b}^{\prime} d x\right) e^{i \omega_{b} T_{0}} \\
& \left.\left.+2 A_{a} A_{b}^{2}\left(\sum_{r=0}^{n} \int_{\eta_{r}}^{\eta_{r+1}} Y_{(m+1), a}^{\prime} Y_{(m+1), b}^{\prime} d x\right) e^{i\left(\omega_{a}+2 \omega_{b}\right) T_{0}}\right] Y_{(m+1), b}^{\prime \prime}\right]+c c+N S T .
\end{aligned}
$$




\subsubsection{Combination resonances of sum type}

One can take two dominant modes (i.e., the $a$ th and $b$ th modes)

$\Omega=\omega_{a}+\omega_{b}+\varepsilon \sigma$

and obtain the solvability conditions as follows:

$$
\begin{aligned}
& D_{1} A_{a}+\frac{\mu}{2} A_{a}+k_{0_{a b}} \bar{A}_{b} e^{i \sigma T_{1}}-k_{3 a b} A_{a}^{2} \bar{A}_{b} \\
& -k_{2_{a b}} A_{a} A_{b} \bar{A}_{b}=0, \\
& D_{1} A_{b}+\frac{\mu}{2} A_{b}+k_{0_{b a}} \bar{A}_{a} e^{i \sigma T_{1}}-k_{3_{b a}} A_{b}^{2} \bar{A}_{b} \\
& -k_{2_{b a}} A_{a} \bar{A}_{a} A_{b}=0,
\end{aligned}
$$

where

$$
\begin{aligned}
& k_{0 a b}=v_{1} \frac{\left[\left(\frac{\Omega}{2}-\omega_{b}\right)\left(\sum_{r=0}^{n} \int_{\eta_{r}}^{\eta_{r+1}} \bar{Y}_{(m+1), b}^{\prime} \bar{Y}_{(m+1), a} d x\right)-i v_{0}\left(\sum_{r=0}^{n} \int_{\eta_{r}}^{\eta_{r+1}} \bar{Y}_{(m+1), b}^{\prime \prime} \bar{Y}_{(m+1), a} d x\right)\right]}{2\left[i \omega_{a}\left(\sum_{r=0}^{n} \int_{\eta_{r}+1}^{\eta_{r+1}} Y_{(m+1), a} \bar{Y}_{(m+1), a} d x\right)+v_{0}\left(\sum_{r=0}^{n} \int_{\eta_{r}}^{\eta_{r+1}} Y_{(m+1), a}^{\prime} \bar{Y}_{(m+1), a} d x\right)\right]}, \\
& k_{0_{b a}}=v_{1} \frac{\left[\left(\frac{\Omega}{2}-\omega_{a}\right)\left(\sum_{r=0}^{n} \int_{\eta_{r}}^{\eta_{r+1}} \bar{Y}_{(m+1), a}^{\prime} \bar{Y}_{(m+1), b} d x\right)-i v_{0}\left(\sum_{r=0}^{n} \int_{\eta_{r}}^{\eta_{r+1}} \bar{Y}_{(m+1), a}^{\prime \prime} \bar{Y}_{(m+1), b} d x\right)\right]}{2\left[i \omega_{b}\left(\sum_{r=0}^{n} \int_{\eta_{r}+1}^{\eta_{r}} Y_{(m+1), b} \bar{Y}_{(m+1), b} d x\right)+v_{0}\left(\sum_{r=0}^{n} \int_{\eta_{r}}^{\eta_{r+1}} Y_{(m+1), b}^{\prime} \bar{Y}_{(m+1), b} d x\right)\right]}, \\
& k_{2}=\frac{1}{2} v_{b}^{2} \frac{\left[2 \sum_{r=0}^{n}\left(\int_{\eta_{r}}^{\eta_{r+1}} Y_{(m+1), a}^{\prime \prime} \bar{Y}_{(m+1), a} d x\right)\left(\int_{\eta_{r}}^{\eta_{r+1}} Y_{(m+1), b}^{\prime} \bar{Y}_{(m+1), b}^{\prime} d x\right)+2 \sum_{r=0}^{n}\left(\int_{\eta_{r}}^{\eta_{r+1}} \bar{Y}_{(m+1), b}^{\prime \prime} \bar{Y}_{(m+1), a} d x\right)\left(\int_{\eta r}^{\eta_{r+1}} Y_{(m+1), a}^{\prime} Y_{(m+1), b}^{\prime} d x\right)\right]}{2\left[i \omega_{a}\left(\sum_{r=0}^{n} \int_{\eta_{r}}^{\eta_{r+1}} Y_{(m+1), a} \bar{Y}_{(m+1), a} d x\right)+v_{0}\left(\sum_{r=0}^{n} \int_{\eta_{r}+1}^{\eta_{r+1}} Y_{(m+1), a}^{\prime} \bar{Y}_{(m+1), a} d x\right)\right]}, \\
& k_{2}=\frac{1}{2} v_{b}^{2} \frac{\left[2 \sum_{r=0}^{n}\left(\int_{\eta_{r}}^{\eta_{r+1}} Y_{(m+1), b}^{\prime \prime} \bar{Y}_{(m+1), b} d x\right)\left(\int_{\eta_{r}}^{\eta_{r+1}} Y_{(m+1), a}^{\prime} \bar{Y}_{(m+1), a}^{\prime} d x\right)+2 \sum_{r=0}^{n}\left(\int_{\eta_{r}}^{\eta_{r+1}} \bar{Y}_{(m+1), a}^{\prime \prime} \bar{Y}_{(m+1), b} d x\right)\left(\int_{\eta_{r}}^{\eta_{r+1}} Y_{(m+1), a}^{\prime} Y_{(m+1), b}^{\prime} d x\right)\right]}{2\left[i \omega_{b}\left(\sum_{r=0}^{n} \int_{\eta_{r}}^{\eta_{r+1}} Y_{(m+1), b} \bar{Y}_{(m+1), b} d x\right)+v_{0}\left(\sum_{r=0}^{n} \int_{\eta_{r}}^{\eta_{r+1}} Y_{(m+1), b}^{\prime} \bar{Y}_{(m+1), b} d x\right)\right]}, \\
& k_{3}=\frac{1}{2} v_{b}^{2} \frac{\left[\sum_{r=0}^{n}\left(\int_{\eta_{r}}^{\eta_{r+1}} \bar{Y}_{(m+1), a}^{\prime \prime} \bar{Y}_{(m+1), a} d x\right)\left(\int_{\eta_{r}}^{\eta_{r+1}} Y_{(m+1), a}^{\prime 2} d x\right)+2 \sum_{r=0}^{n}\left(\int_{\eta_{r}}^{\eta_{r+1}} Y_{(m+1), a}^{\prime \prime} \bar{Y}_{(m+1), a} d x\right)\left(\int_{\eta_{r}}^{\eta_{r+1}} Y_{(m+1), a}^{\prime} \bar{Y}_{(m+1), a}^{\prime} d x\right)\right]}{2\left[i \omega_{a}\left(\sum_{r=0}^{n} \int_{\eta_{r}+1}^{\eta_{r}} Y_{(m+1), a} \bar{Y}_{(m+1), a} d x\right)+v_{0}\left(\sum_{r=0}^{n} \int_{\eta_{r}}^{\eta_{r+1}} Y_{(m+1), a}^{\prime} \bar{Y}_{(m+1), a} d x\right)\right]}, \\
& k_{3 b a}=\frac{1}{2} v_{b}^{2} \frac{\left[\sum_{r=0}^{n}\left(\int_{\eta_{r}}^{\eta_{r+1}} \bar{Y}_{(m+1), b}^{\prime \prime} \bar{Y}_{(m+1), b} d x\right)\left(\int_{\eta_{r}}^{\eta_{r+1}} Y_{(m+1), a}^{\prime 2} d x\right)+2 \sum_{r=0}^{n}\left(\int_{\eta_{r}}^{\eta_{r+1}} Y_{(m+1), b}^{\prime \prime} \bar{Y}_{(m+1), b} d x\right)\left(\int_{\eta_{r}}^{\eta_{r+1}} Y_{(m+1), b}^{\prime} \bar{Y}_{(m+1), b}^{\prime} d x\right)\right]}{2\left[i \omega_{b}\left(\sum_{r=0}^{n} \int_{\eta_{r}+1}^{\eta_{r}} Y_{(m+1), b} \bar{Y}_{(m+1), b} d x\right)+v_{0}\left(\sum_{r=0}^{n} \int_{\eta_{r}}^{\eta_{r}+1} Y_{(m+1), b}^{\prime} \bar{Y}_{(m+1), b} d x\right)\right]} .
\end{aligned}
$$

To solve Eqs. (52) and (53), we assume the form

$$
A_{a}=\frac{1}{2} a_{a}\left(T_{1}\right) e^{i \theta_{a}\left(T_{1}\right)}, \quad A_{b}=\frac{1}{2} a_{b}\left(T_{1}\right) e^{i \theta_{b}\left(T_{1}\right)}
$$

and obtain

$$
\begin{aligned}
& D_{1} a_{a}+a_{a} i D_{1} \theta_{a}+\frac{\mu}{2} a_{a}+k_{0_{a b}} a_{b} e^{i \gamma} \\
& -\frac{1}{4} k_{3_{a b}} a_{a}^{3}-\frac{1}{4} k_{2_{a b}} a_{a} a_{b}^{2}=0, \\
& D_{1} a_{b}+a_{b} i D_{1} \theta_{b}+\frac{\mu}{2} a_{b}+k_{0_{b a}} a_{a} e^{i \gamma}-\frac{1}{4} k_{3 b a} a_{b}^{3} \\
& -\frac{1}{4} k_{2_{b a}} a_{a}^{2} a_{b}=0 .
\end{aligned}
$$

We can make another transformation using $\gamma=$ $\sigma T_{1}-\theta_{a}-\theta_{b}$. Since the complex coefficients in
Eq. (53) are $k_{0 a b}=k_{0 a b_{R}}+i k_{0 a b_{\mathrm{I}}}, k_{2 a b}=i k_{2 a b_{\mathrm{I}}}$, $k_{3 a b}=i k_{3 a b_{\mathrm{I}}}, k_{0 b a}=k_{0 b a_{R}}+i k_{0 b a_{\mathrm{I}}}, k_{2 b a}=i k_{2 b a_{\mathrm{I}}}$, and $k_{3 b a}=i k_{3 b a_{\mathrm{I}}}$, we obtain the following amplitude modulation equations:

$$
\begin{aligned}
D_{1} a_{a}= & -\frac{\mu}{2} a_{a}+k_{0 a b_{\mathrm{I}}} a_{b} \sin \gamma-k_{0 a b_{R}} a_{b} \cos \gamma=F_{1}, \\
D_{1} a_{b}= & -\frac{\mu}{2} a_{b}+k_{0 b a_{\mathrm{I}}} a_{a} \sin \gamma-k_{0 b a_{R}} a_{a} \cos \gamma=F_{2}, \\
D_{1} \gamma= & \sigma+\frac{1}{a_{a} a_{b}}\left(k_{0 a b_{R}} a_{b}^{2}+k_{0 b a_{R}} a_{a}^{2}\right) \sin \gamma \\
& +\frac{1}{a_{a} a_{b}}\left(k_{0 a b_{\mathrm{I}}} a_{b}^{2}+k_{0 b a_{\mathrm{I}}} a_{a}^{2}\right) \cos \gamma \\
& -\frac{a_{a}^{2}}{4}\left(k_{3 a b_{\mathrm{I}}}+k_{2 b a_{\mathrm{I}}}\right)-\frac{a_{b}^{2}}{4}\left(k_{2 a b_{\mathrm{I}}}+k_{3 b a_{\mathrm{I}}}\right)=F_{3} .
\end{aligned}
$$

Now we can consider the steady-state response $D_{1} a_{a}=$ $0, D_{1} a_{b}=0, D_{1} \gamma=0$. Negative real parts of the eigenvalues obtained from the Jacobian matrix shown 
below are stable. The positive roots of the real parts are unstable.

$$
\left[\begin{array}{lll}
\frac{\partial F_{1}}{\partial a_{a}} & \frac{\partial F_{1}}{\partial a_{b}} & \frac{\partial F_{1}}{\partial \gamma} \\
\frac{\partial F_{2}}{\partial a_{a}} & \frac{\partial F_{2}}{\partial a_{b}} & \frac{\partial F_{2}}{\partial \gamma} \\
\frac{\partial F_{3}}{\partial a_{a}} & \frac{\partial F_{3}}{\partial a_{b}} & \frac{\partial F_{3}}{\partial \gamma}
\end{array}\right] \begin{gathered}
\\
\begin{array}{c}
a_{a}=a_{0 a} \\
a_{b}=a_{0 b} \\
\gamma=\gamma_{0}
\end{array}
\end{gathered} .
$$

One can write complex amplitudes in polar form to perform a stability analysis for the trivial solutions

$$
\begin{aligned}
& A_{a}=\frac{1}{2}\left(p_{a}+i p_{a}\right) e^{i \frac{\sigma}{2} T_{1}}, \\
& A_{b}=\frac{1}{2}\left(p_{b}+i p_{b}\right) e^{i \frac{\sigma}{2} T_{1}}
\end{aligned}
$$

and obtain the following amplitude phase modulation equations:

$$
\begin{aligned}
D_{1} p_{a}= & q_{a}\left(\frac{\sigma}{2}+\frac{1}{4} k_{3 a b_{\mathrm{I}}}\left(p_{a}^{2}-q_{a}^{2}\right)\right. \\
& \left.+\frac{1}{4} k_{2 a b_{\mathrm{I}}}\left(p_{b}^{2}+q_{b}^{2}\right)\right) \\
& -\frac{\mu}{2} p_{a}-k_{0 a b_{R}} p_{b}-k_{0 a b_{\mathrm{I}}} q_{b}=f_{1}, \\
D_{1} p_{b}= & q_{b}\left(\frac{\sigma}{2}+\frac{1}{4} k_{3 b a_{\mathrm{I}}}\left(p_{b}^{2}-q_{b}^{2}\right)\right. \\
& \left.+\frac{1}{4} k_{2 b a_{\mathrm{I}}}\left(p_{a}^{2}+q_{a}^{2}\right)\right) \\
& -\frac{\mu}{2} p_{b}-k_{0 b a_{R}} p_{a}-k_{0 b a_{\mathrm{I}}} q_{a}=f_{2}, \\
D_{1} q_{a}= & p_{a}\left(-\frac{\sigma}{2}+\frac{1}{4} k_{3 a b_{\mathrm{I}}}\left(p_{a}^{2}+q_{a}^{2}\right)\right. \\
& \left.+\frac{1}{4} k_{2 a b_{\mathrm{I}}}\left(p_{b}^{2}+q_{b}^{2}\right)\right) \\
& -\frac{\mu}{2} q_{a}-k_{0 a b_{R}} q_{b}-k_{0 a b_{\mathrm{I}}} p_{b}=f_{3},
\end{aligned}
$$

$$
\begin{aligned}
k_{4_{a b}}= & v_{1} \frac{\left[-\left(\omega_{b}+\frac{\Omega}{2}\right)\left(\sum_{r=0}^{n} \int_{\eta_{r}}^{\eta_{r+1}} Y_{(m+1), b}^{\prime} \bar{Y}_{(m+1), a} d x\right)+i v_{0}\left(\sum_{r=0}^{n} \int_{\eta_{r}}^{\eta_{r+1}} Y_{(m+1), b}^{\prime \prime} \bar{Y}_{(m+1), a} d x\right)\right]}{2\left[i \omega_{a}\left(\sum_{r=0}^{n} \int_{\eta_{r}}^{\eta_{r+1}} Y_{(m+1), a} \bar{Y}_{(m+1), a} d x\right)+v_{0}\left(\sum_{r=0}^{n} \int_{\eta_{r}}^{\eta_{r+1}} Y_{(m+1), a}^{\prime} \bar{Y}_{(m+1), a} d x\right)\right]}, \\
k_{5_{a b}=} & \frac{1}{2} v_{b}^{2}\left[\left(\sum_{r=0}^{n} \int_{\eta_{r}}^{\eta_{r+1}} Y_{(m+1), a}^{\prime \prime} \bar{Y}_{(m+1), a} d x\right)\left(\sum_{r=0}^{n} \int_{\eta_{r}}^{\eta_{r+1}} Y_{(m+1), b}^{\prime} \bar{Y}_{(m+1), b} d x\right)\right. \\
& +\left(\sum_{r=0}^{n} \int_{\eta_{r}}^{\eta_{r+1}} \bar{Y}_{(m+1), b}^{\prime \prime} \bar{Y}_{(m+1), a} d x\right)\left(\sum_{r=0}^{n} \int_{\eta_{r}}^{\eta_{r+1}} Y_{(m+1), a}^{\prime} Y_{(m+1), b}^{\prime} d x\right)
\end{aligned}
$$

where suming that $a>b$ without loss of generality:

$\Omega=\omega_{a}-\omega_{b}+\varepsilon \sigma$. are obtained:

$$
\begin{aligned}
& D_{1} A_{a}+\frac{\mu}{2} A_{a}-k_{4_{a b}} A_{b} e^{i \sigma T_{1}}-k_{3_{a b}} A_{a}^{2} \bar{A}_{b} \\
& \quad-k_{5_{a b}} A_{a} A_{b} \bar{A}_{b}=0, \\
& D_{1} A_{b}+\frac{\mu}{2} A_{b}-k_{6_{b a}} A_{a} e^{-i \sigma T_{1}}-k_{3_{b a}} A_{b}^{2} \bar{A}_{b} \\
& \quad-k_{7_{b a}} A_{a} \bar{A}_{a} A_{b}=0,
\end{aligned}
$$

Now let us investigate combination resonances of difference type. The velocity variation may be nearly equal to the difference of the $a$ th and $b$ th modes as-

Similarly, the following complex amplitude equations 


$$
\begin{aligned}
+ & \left.\left(\sum_{r=0}^{n} \int_{\eta_{r}}^{\eta_{r+1}} Y_{(m+1), b}^{\prime \prime} \bar{Y}_{(m+1), a} d x\right)\left(\sum_{r=0}^{n} \int_{\eta_{r}}^{\eta_{r+1}} Y_{(m+1), a}^{\prime} \bar{Y}_{(m+1), b}^{\prime} d x\right)\right] \\
& /\left[i \omega_{a}\left(\sum_{r=0}^{n} \int_{\eta_{r}}^{\eta_{r+1}} Y_{(m+1), a} \bar{Y}_{(m+1), a} d x\right)+v_{0}\left(\sum_{r=0}^{n} \int_{\eta_{r}}^{\eta_{r+1}} Y_{(m+1), a}^{\prime} \bar{Y}_{(m+1), a} d x\right)\right], \\
k_{b_{b a}=}= & v_{1} \frac{\left[\left(\omega_{a}-\frac{\Omega}{2}\right)\left(\sum_{r=0}^{n} \int_{\eta_{r}}^{\eta_{r+1}} Y_{(m+1), a}^{\prime} \bar{Y}_{(m+1), b} d x\right)-i v_{0}\left(\sum_{r=0}^{n} \int_{\eta_{r}}^{\eta_{r+1}} Y_{(m+1), a}^{\prime \prime} \bar{Y}_{(m+1), b} d x\right)\right]}{\left.\omega_{b}\left(\sum_{r=0}^{n} \int_{\eta_{r}}^{\eta_{r+1}} Y_{(m+1), b} \bar{Y}_{(m+1), b} d x\right)+v_{0}\left(\sum_{r=0}^{n} \int_{\eta_{r}}^{\eta_{r+1}} Y_{(m+1), b}^{\prime} \bar{Y}_{(m+1), b} d x\right)\right]} \\
k_{7_{b a}=} & \frac{1}{2} v_{b}^{2}\left[\left(\sum_{r=0}^{n} \int_{\eta_{r}}^{\eta_{r+1}} Y_{(m+1), b}^{\prime \prime} \bar{Y}_{(m+1), b} d x\right)\left(\sum_{r=0}^{n} \int_{\eta_{r}}^{\eta_{r+1}} Y_{(m+1), a}^{\prime} \bar{Y}_{(m+1), a} d x\right)\right. \\
+ & \left(\sum_{r=0}^{n} \int_{\eta_{r}}^{\eta_{r+1}} \bar{Y}_{(m+1), a}^{\prime \prime} \bar{Y}_{(m+1), b} d x\right)\left(\sum_{r=0}^{n} \int_{\eta_{r}}^{\eta_{r+1}} Y_{(m+1), a}^{\prime} Y_{(m+1), b}^{\prime} d x\right) \\
+ & \left.\left(\sum_{r=0}^{n} \int_{\eta_{r}}^{\eta_{r+1}} Y_{(m+1), a}^{\prime \prime} \bar{Y}_{(m+1), b} d x\right)\left(\sum_{r=0}^{n} \int_{\eta_{r}}^{\eta_{r+1}} Y_{(m+1), a}^{\prime} \bar{Y}_{(m+1), b}^{\prime} d x\right)\right] \\
& /\left[i \omega_{b}\left(\sum_{r=0}^{n} \int_{\eta_{r}}^{\eta_{r+1}} Y_{(m+1), b} \bar{Y}_{(m+1), b} d x\right)+v_{0}\left(\sum_{r=0}^{n} \int_{\eta_{r}}^{\eta_{r+1}} Y_{(m+1), b}^{\prime} \bar{Y}_{(m+1), b} d x\right)\right] .
\end{aligned}
$$

The following transformation can be used:

$$
A_{a}=\frac{1}{2} a_{a}\left(T_{1}\right) e^{-i \theta_{a}\left(T_{1}\right)}, \quad A_{b}=\frac{1}{2} a_{b}\left(T_{1}\right) e^{i \theta_{b}\left(T_{1}\right)} .
$$

Equations (60)-(61) become

$$
\begin{gathered}
D_{1} a_{a}-a_{a} i D_{1} \theta_{a}+\frac{\mu}{2} a_{a}-k_{4 a b} a_{b} e^{i \gamma}-\frac{1}{4} k_{3 a b} a_{a}^{3} \\
-\frac{1}{4} k_{5_{a b}} a_{a} a_{b}^{2}=0,
\end{gathered}
$$$$
D_{1} a_{b}+a_{b} i D_{1} \theta_{b}+\frac{\mu}{2} a_{b}-k_{6_{b a}} a_{a} e^{-i \gamma}-\frac{1}{4} k_{3 b a} a_{b}^{3}
$$$$
-\frac{1}{4} k_{7_{b a}} a_{a}^{2} a_{b}=0 \text {. }
$$

Further, the transformation using $\gamma=\sigma T_{1}+\theta_{a}+$ $\theta_{b}$ shows that the coefficients have only imaginary parts, $k_{3 a b}=i k_{3 a b_{\mathrm{I}}}, k_{4 a b}=k_{4 a b_{R}}+i k_{4 a b_{\mathrm{I}}}, k_{5 a b}=$ $i k_{5 a b_{\mathrm{I}}}, k_{6 b a}=k_{6 b a_{R}}+i k_{6 b a_{\mathrm{I}}}, k_{7 b a}=i k_{7 b a_{\mathrm{I}}}$, and the amplitude-phase modulations can be written as follows:

$D_{1} a_{a}=-\frac{\mu}{2} a_{a}-k_{4 a b_{\mathrm{I}}} a_{b} \sin \gamma+k_{4 a b_{R}} a_{b} \cos \gamma=F_{1}$,

$D_{1} a_{b}=-\frac{\mu}{2} a_{b}+k_{6 b a_{\mathrm{I}}} a_{a} \sin \gamma+k_{6 b a_{R}} a_{a} \cos \gamma=F_{2}$,

$$
\begin{aligned}
D_{1} \gamma= & \sigma-\frac{1}{a_{a} a_{b}}\left(k_{4 a b_{R}} a_{b}^{2}+k_{6 b a_{R}} a_{a}^{2}\right) \sin \gamma \\
& -\frac{1}{a_{a} a_{b}}\left(k_{4 a b_{\mathrm{I}}} a_{b}^{2}-k_{6 b a_{\mathrm{I}}} a_{a}^{2}\right) \cos \gamma \\
& -\frac{a_{a}^{2}}{4}\left(k_{3 a b_{\mathrm{I}}}-k_{7 b a_{\mathrm{I}}}\right)-\frac{a_{b}^{2}}{4}\left(k_{5 a b_{\mathrm{I}}}-k_{3 b a_{\mathrm{I}}}\right)=F_{3} .
\end{aligned}
$$

At steady state, $D_{1} a_{a}=0, D_{1} a_{b}=0$, and $D_{1} \gamma=0$. Inserting into the Jacobian matrix

$$
\left[\begin{array}{lll}
\frac{\partial F_{1}}{\partial a_{a}} & \frac{\partial F_{1}}{\partial a_{b}} & \frac{\partial F_{1}}{\partial \gamma} \\
\frac{\partial F_{2}}{\partial a_{a}} & \frac{\partial F_{2}}{\partial a_{b}} & \frac{\partial F_{2}}{\partial \gamma} \\
\frac{\partial F_{3}}{\partial a_{a}} & \frac{\partial F_{3}}{\partial a_{b}} & \frac{\partial F_{3}}{\partial \gamma}
\end{array}\right] \begin{gathered}
\\
\begin{array}{r}
a_{a}=a_{0 a} \\
a_{b}=a_{0 b} \\
\gamma=\gamma_{0}
\end{array}
\end{gathered},
$$

one can search for stability of the solutions.

The stability of trivial solutions can be obtained similarly. We start by writing the complex amplitudes in the polar form

$$
\begin{aligned}
& A_{a}=\frac{1}{2}\left(p_{a}+i q_{a}\right) e^{i \frac{\sigma}{2} T_{1}}, \\
& A_{b}=\frac{1}{2}\left(p_{b}+i q_{b}\right) e^{-i \frac{\sigma}{2} T_{1}},
\end{aligned}
$$




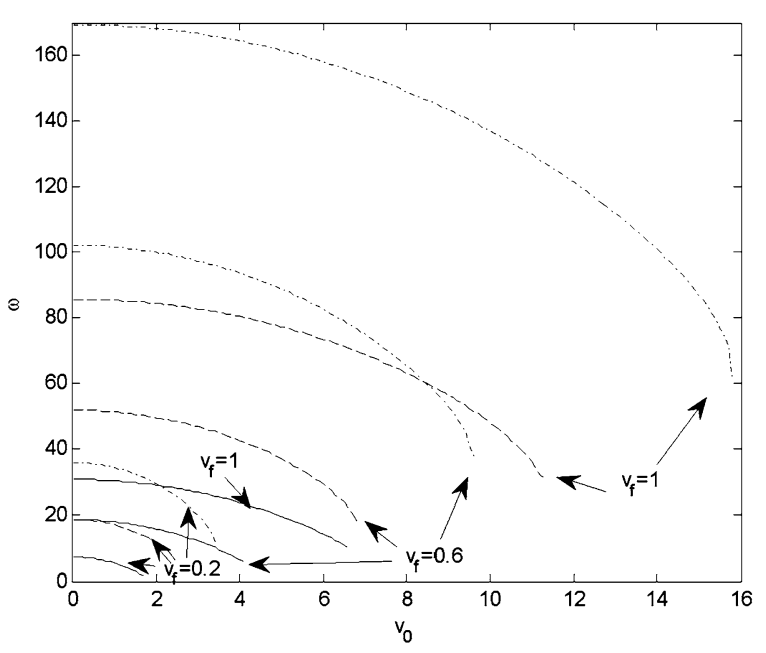

Fig. 2 Variations of the first three modes with axial mean velocity for locations $\eta_{1}=0.1$ and $\eta_{2}=0.9$ and for different $v_{f}$ values $\left(\omega_{1}:-, \omega_{2}:---, \omega_{3}:-\cdot-\right)$

and inserting into Eqs. (60) and (61), we obtain

$D_{1} p_{a}$

$$
\begin{aligned}
= & q_{a}\left(\frac{\sigma}{2}-\frac{1}{4} k_{3 a b_{\mathrm{I}}}\left(p_{a}^{2}+q_{a}^{2}\right)-\frac{1}{4} k_{5 a b_{\mathrm{I}}}\left(p_{b}^{2}+q_{b}^{2}\right)\right) \\
- & \frac{\mu}{2} p_{a}+k_{4 a b_{R}} p_{b}-k_{4 a b_{\mathrm{I}}} q_{b}=f_{1},
\end{aligned}
$$

$D_{1} p_{b}$

$$
\begin{gathered}
=q_{b}\left(-\frac{\sigma}{2}-\frac{1}{4} k_{3 b a_{\mathrm{I}}}\left(p_{b}^{2}+q_{b}^{2}\right)-\frac{1}{4} k_{7 b a_{\mathrm{I}}}\left(p_{a}^{2}+q_{a}^{2}\right)\right) \\
-\frac{\mu}{2} p_{b}+k_{6 b a_{R}} p_{a}-k_{6 b a_{\mathrm{I}}} q_{a}=f_{2},
\end{gathered}
$$

$D_{1} q_{a}$

$$
\begin{aligned}
= & p_{a}\left(-\frac{\sigma}{2}+\frac{1}{4} k_{3 a b_{\mathrm{I}}}\left(p_{a}^{2}+q_{a}^{2}\right)+\frac{1}{4} k_{5 a b_{\mathrm{I}}}\left(p_{b}^{2}+q_{b}^{2}\right)\right) \\
& -\frac{\mu}{2} q_{a}-k_{4 a b_{R}} q_{b}+k_{4 a b_{\mathrm{I}}} p_{b}=f_{3},
\end{aligned}
$$

$D_{1} q_{b}$

$$
\begin{aligned}
= & p_{b}\left(\frac{\sigma}{2}+\frac{1}{4} k_{3 b a_{\mathrm{I}}}\left(p_{b}^{2}+q_{b}^{2}\right)+\frac{1}{4} k_{7 b a_{\mathrm{I}}}\left(p_{a}^{2}+q_{a}^{2}\right)\right) \\
- & \frac{\mu}{2} q_{b}-k_{6 b a_{R}} q_{a}+k_{6 b a_{\mathrm{I}}} p_{a}=f_{4} .
\end{aligned}
$$

At the steady state, $D_{1} p_{a}=0, D_{1} p_{b}=0, D_{1} q_{a}=0$, $D_{1} q_{b}=0$; then we construct the Jacobian matrix. No instabilities arise up to the second order of expansion for difference type of combination resonances.

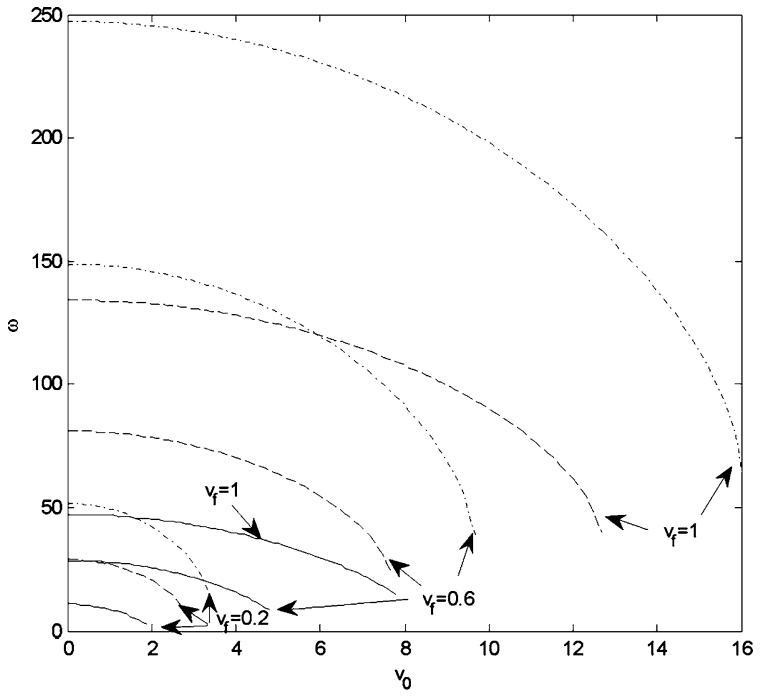

Fig. 3 Variations of the first three modes with axial mean velocity for locations $\eta_{1}=0.2$ and $\eta_{2}=0.8$ and for different $v_{f}$ values $\left(\omega_{1}:-, \omega_{2}:---, \omega_{3}:-\cdot-\right)$

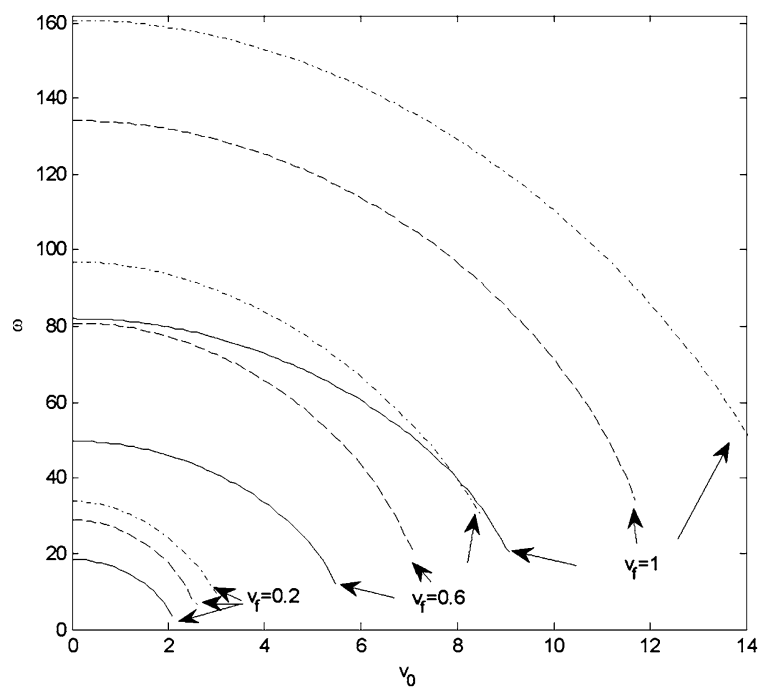

Fig. 4 Variations of the first three modes with axial mean velocity for locations $\eta_{1}=0.3$ and $\eta_{2}=0.7$ and for different $v_{f}$ values $\left(\omega_{1}:-, \omega_{2}:---, \omega_{3}:-\cdot-\right)$

\section{Numerical results}

The solution of these equations for different parameters give variation of frequency with the mean axial velocity. Material and geometric properties of the moving beam are chosen as follows: $E=200 \mathrm{GPa}$, $L=1 \mathrm{~m}, b=0.002 \mathrm{~m}, h=0.001 \mathrm{~m}, \rho=7.8 \mathrm{gr} / \mathrm{m}^{3}$. In Figs. 2, 3, 4 and 5, the variation is depicted for four-support cases $\eta_{1}-\eta_{2}=0.1-0.9, \eta_{1}-\eta_{2}=0.2-$ 


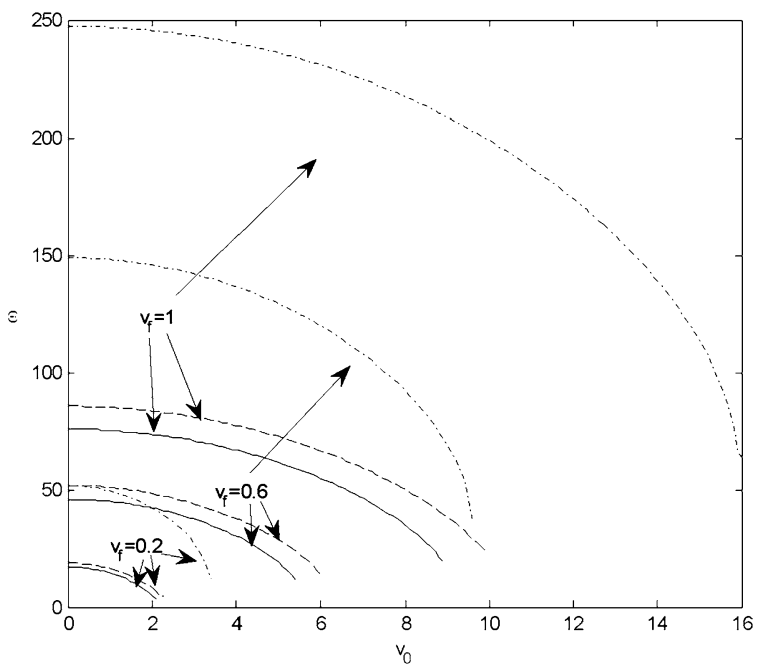

Fig. 5 Variations of the first three modes with axial mean velocity for locations $\eta_{1}=0.4$ and $\eta_{2}=0.6$ and for different $v_{f}$ values $\left(\omega_{1}:-, \omega_{2}:---, \omega_{3}:-\cdot-\right)$

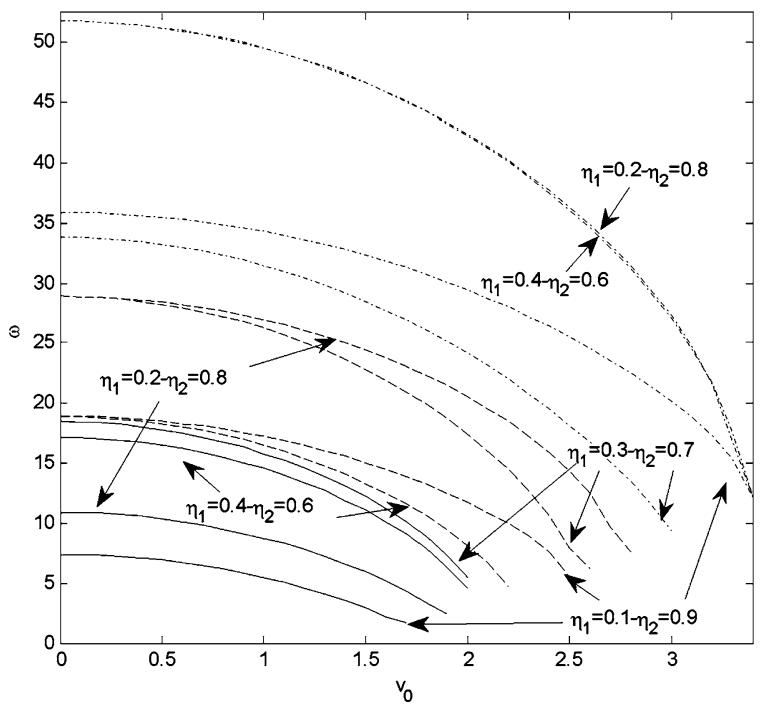

Fig. 6 Variation of the first three modes with axial mean velocity for $v_{f}=0.2$ and for different $\eta_{1}-\eta_{2}$ locations $\left(\omega_{1}:-, \omega_{2}\right.$ :$\left.--, \omega_{3}:-\cdot-\right)$

$0.8, \eta_{1}-\eta_{2}=0.3-0.7$, and $\eta_{1}-\eta_{2}=0.4-0.6$, respectively, for different $v_{f}$ values in the first three natural frequencies. Locating the supports toward the middle section increases the frequencies. Frequencies decrease with an increase in the axial mean velocity. This situation is the characteristic for axially moving systems. Increasing the flexural rigidity constant increases the frequencies as expected. Variations of the first three modes with axial mean velocity for $v_{f}=0.2$

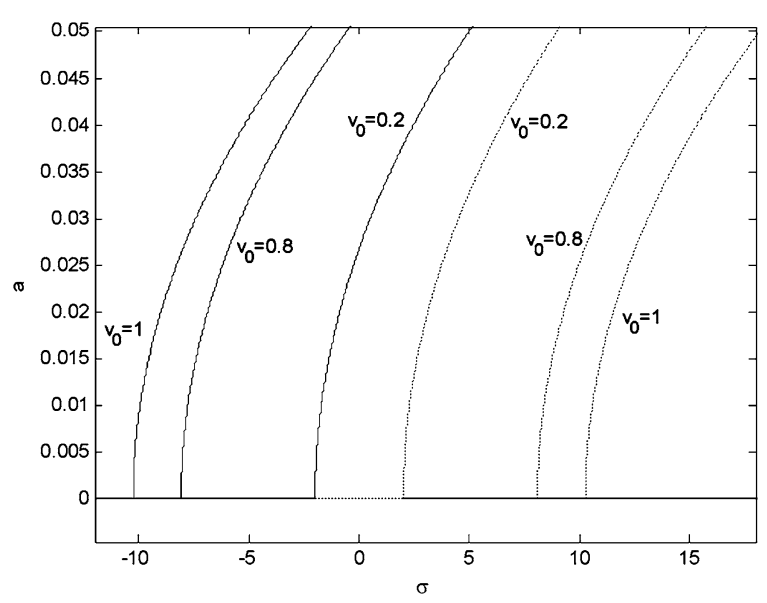

Fig. 7 Nonlinear frequency-amplitude variation for different $v_{0}$ values and for the first mode $\left(v_{f}=0.2, \eta_{1}=0.3, \eta_{2}=0.7\right)$

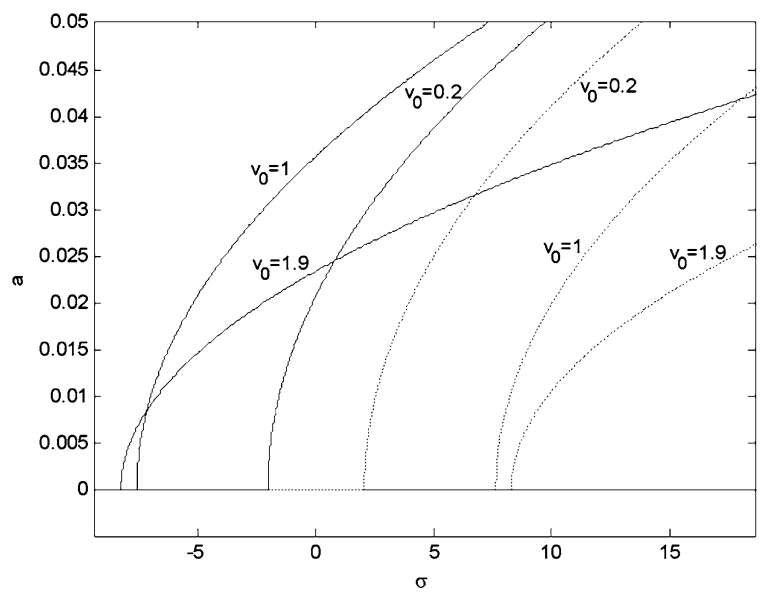

Fig. 8 Nonlinear frequency-amplitude variation for different $v_{0}$ values and for the second mode $\left(v_{f}=0.2, \eta_{1}=0.3\right.$, $\left.\eta_{2}=0.7\right)$

and for different $\eta_{1}-\eta_{2}$ location values are depicted in Fig. 6.

In Fig. 7, $\sigma-a$ variation is depicted for the first mode for $v_{f}=0.2, \eta_{1}-\eta_{2}=0.3-0.7$, and $v_{0}=0.2$, 0.8 , and 1. In Fig. 8, $\sigma-a$ variation is depicted for the second mode, for $v_{f}=0.2, \eta_{1}-\eta_{2}=0.3-0.7$, and $v_{0}=0.2,1$ and 1.9 , respectively. As the mean speed increases, the unstable regions widen. Dashed lines denote unstable solutions. When the intermediate supports are located close to the center, e.g., $\eta=0.3$ and $\eta_{1}-\eta_{2}=0.3-0.7$ as in Figs. 9 and 10 , the unstable regions slightly widen, which can be seen when a comparison made between three-support and four-support cases. All figures are of hardening 
type, but as the intermediate supports are approached to the midpoint, the behavior becomes more hardening.

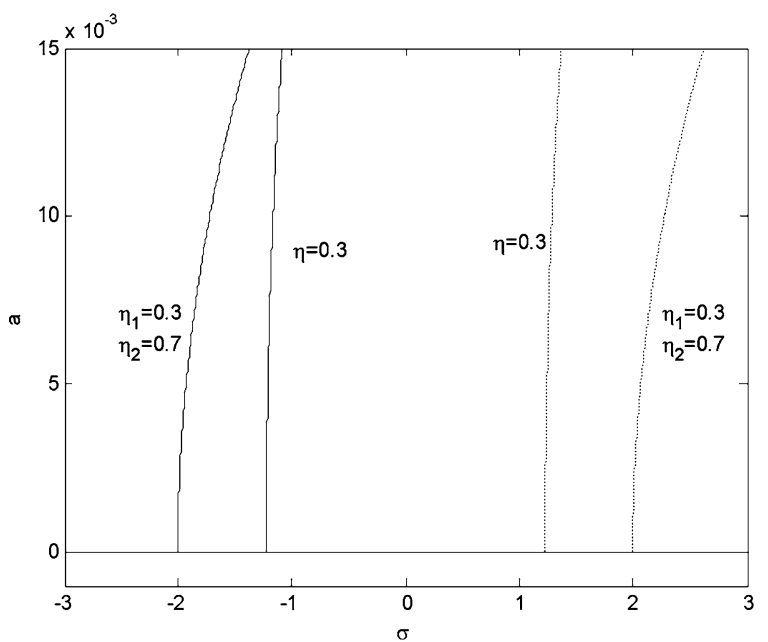

Fig. 9 Nonlinear frequency-amplitude variation for the first mode $\left(v_{f}=0.2, v_{0}=0.2\right)$
In Figs. 11, 12 and 13, variation of stability region depending on mean speed and velocity fluctuation frequency is shown for the first modes of vibration for

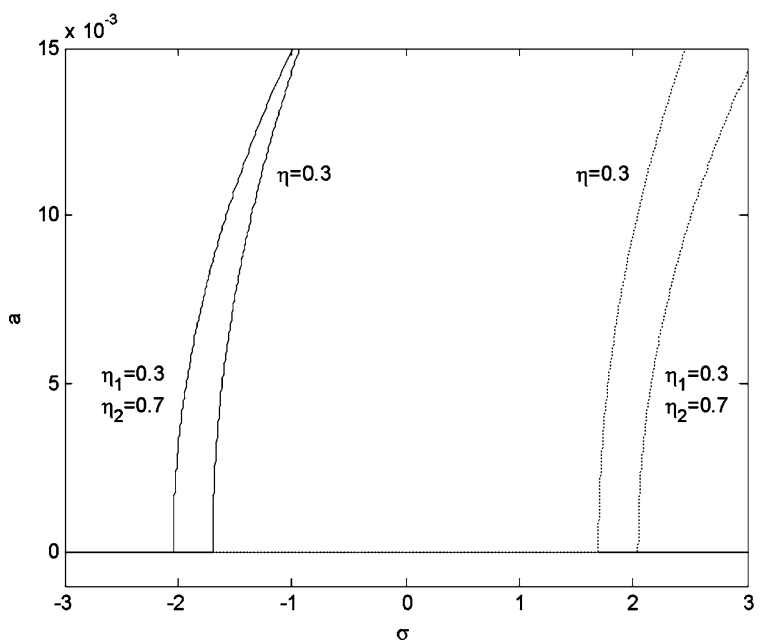

Fig. 10 Nonlinear frequency-amplitude variation for the second mode $\left(v_{f}=0.2, v_{0}=0.2\right)$
Fig. 11 Variation of $\Omega-\varepsilon v_{1}$ values for different $v_{0}$ values and for the first natural frequency

$\left(v_{f}=0.2\right.$,

$\left.\eta_{1}-\eta_{2}=0.3-0.7\right)$

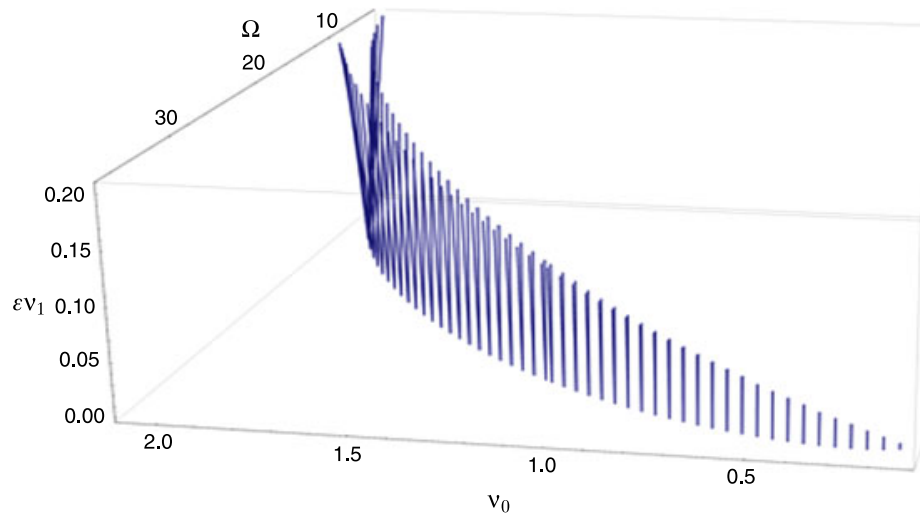

Fig. 12 Variation of $\Omega-\varepsilon v_{1}$ values for different $v_{0}$ values and for the first natural frequency

$\left(v_{f}=0.6\right.$,

$\left.\eta_{1}-\eta_{2}=0.3-0.7\right)$

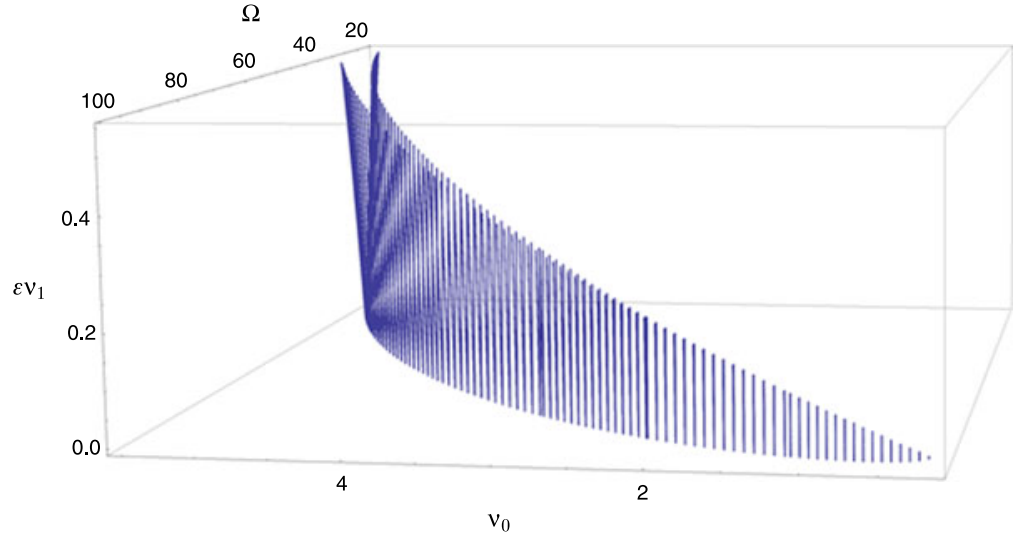


Fig. 13 Variation of $\Omega-\varepsilon v_{1}$ values for different $v_{0}$ values and for the first natural frequency $\left(v_{f}=1\right.$, $\left.\eta_{1}-\eta_{2}=0.3-0.7\right)$
Fig. 14 Variation of $\Omega-\varepsilon v_{1}$ values for different $v_{0}$ values and for the second natural frequency

$\left(v_{f}=0.6\right.$,

$\left.\eta_{1}-\eta_{2}=0.3-0.7\right)$
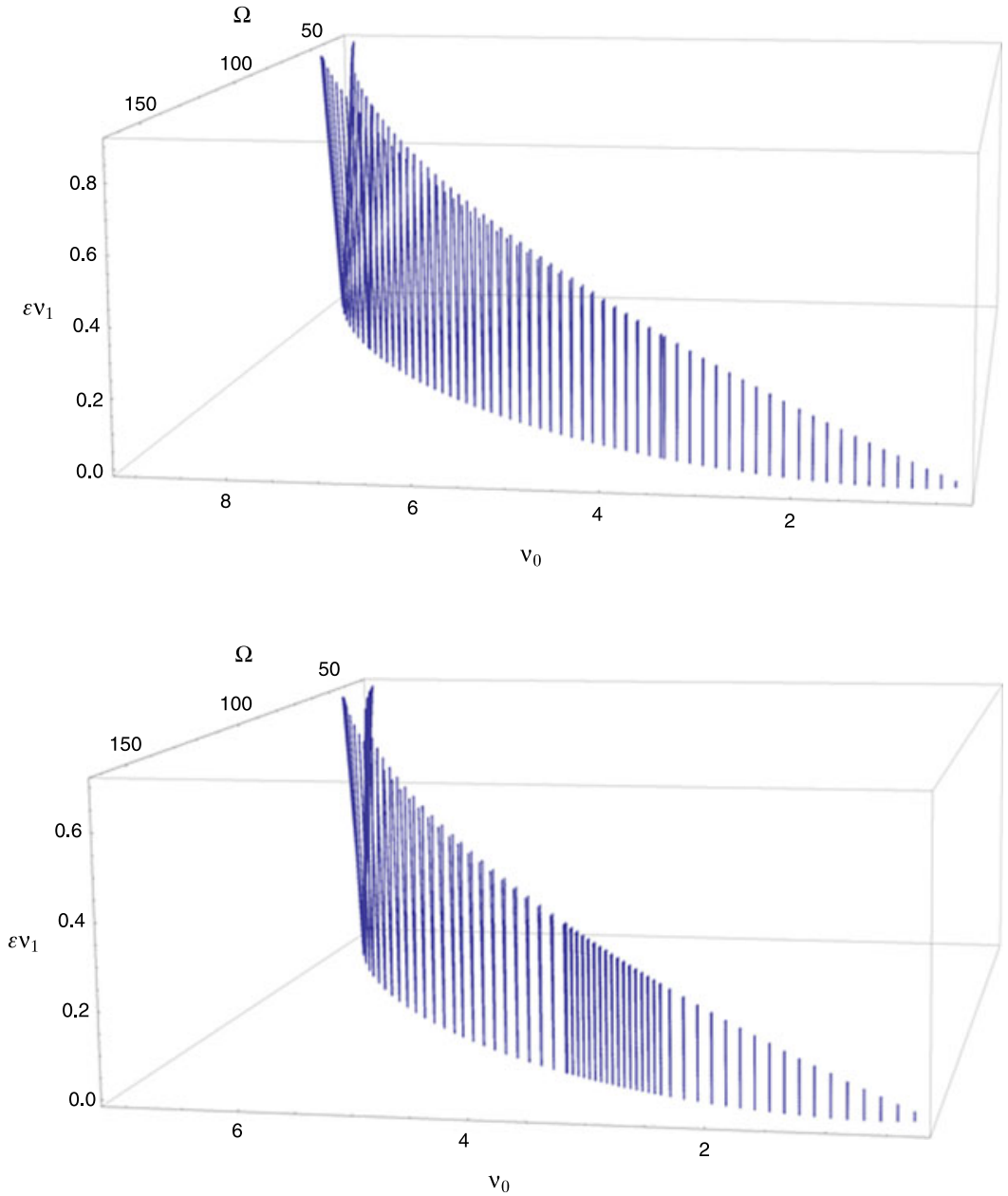

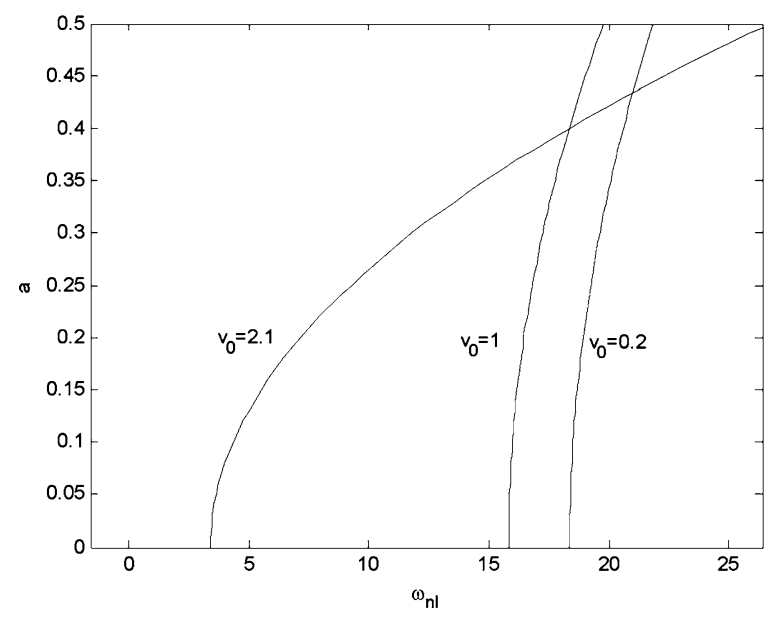

Fig. 15 Nonlinear frequency-amplitude variation for $v_{f}=0.2$ and support locations $\eta_{1}=0.3, \eta_{2}=0.7$ (the first mode) $v_{f}=0.2,0.6$, and 1.0 , respectively. The support locations are the same and only four-support case is discussed. For the same $v_{f}$ (e.g., 0.2), the stability regions become wider with increasing mean speed. The second mode for $v_{f}=0.6$ and $\eta_{1}-\eta_{2}=0.3-0.7$ is shown in Fig. 14 as an example for higher modes only.

In Figs. 15, 16 and 17, the nonlinear frequency versus amplitude is depicted for $v_{f}=0.2, \eta_{1}-\eta_{2}=0.3-$ 0.7 , for different mean speed values, and for the first three modes, respectively. A hardening type behavior is shown in all figures. Increasing the mean speed reduces nonlinear frequencies, but the behavior becomes more hardening. Similar variations for different support locations are presented in Figs. 18, 19 and 20 for the four-support case only. The support location has different effects for different modes due to closeness to the nodal points. 


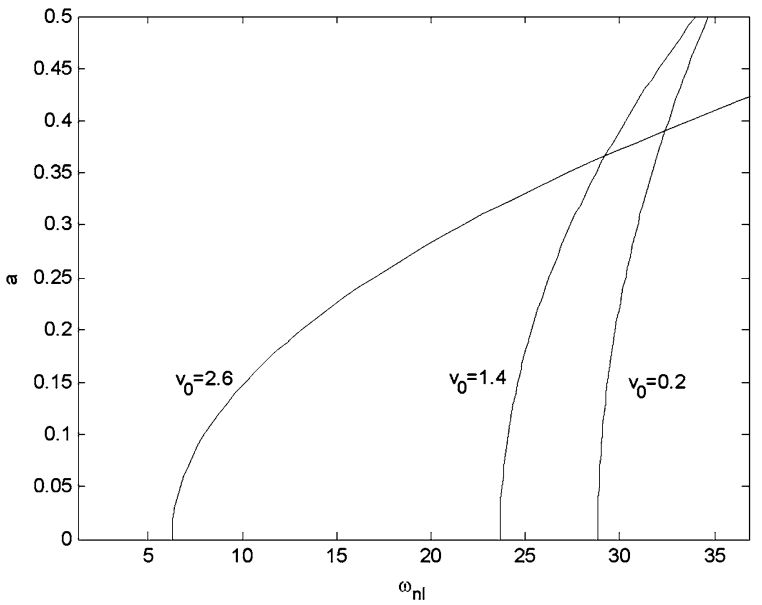

Fig. 16 Nonlinear frequency-amplitude variation for $v_{f}=0.2$ and support locations $\eta_{1}=0.3, \eta_{2}=0.7$ (the second mode)

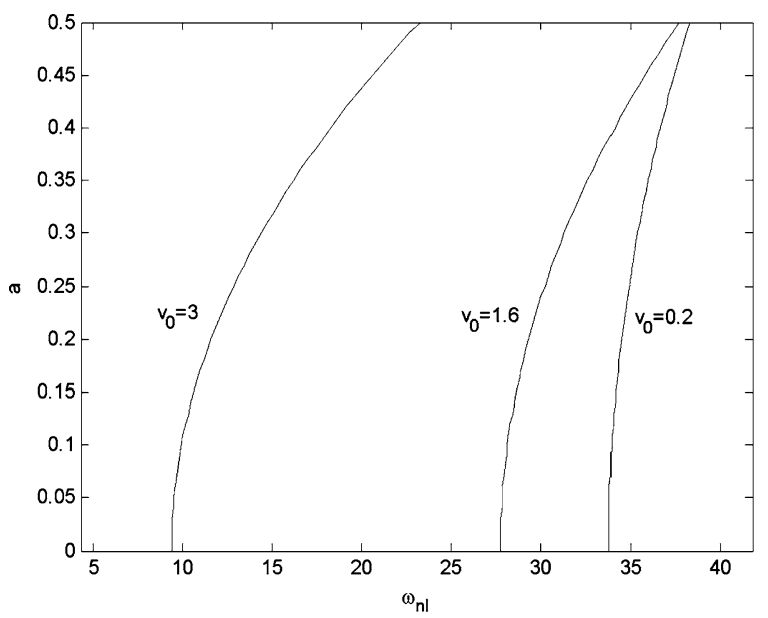

Fig. 17 Nonlinear frequency-amplitude variation for $v_{f}=0.2$ and support locations $\eta_{1}=0.3, \eta_{2}=0.7$ (the third mode)

\section{Conclusions}

Transverse vibrations of an axial moving beam are examined. Equation of motion for an arbitrary number of supports and extension of neutral axis is obtained. The method of multiple scales is applied to these equations. The effects of supports, axial speed, and flexural rigidity on frequencies are discussed. A support condition whose determinant gives eigenvalues for arbitrary number of supports is presented in a general form. Principal parametric resonances and combination resonances are investigated for the frequencies twice the velocity fluctuation frequency. The general form of frequency equation is presented in a matrix form for

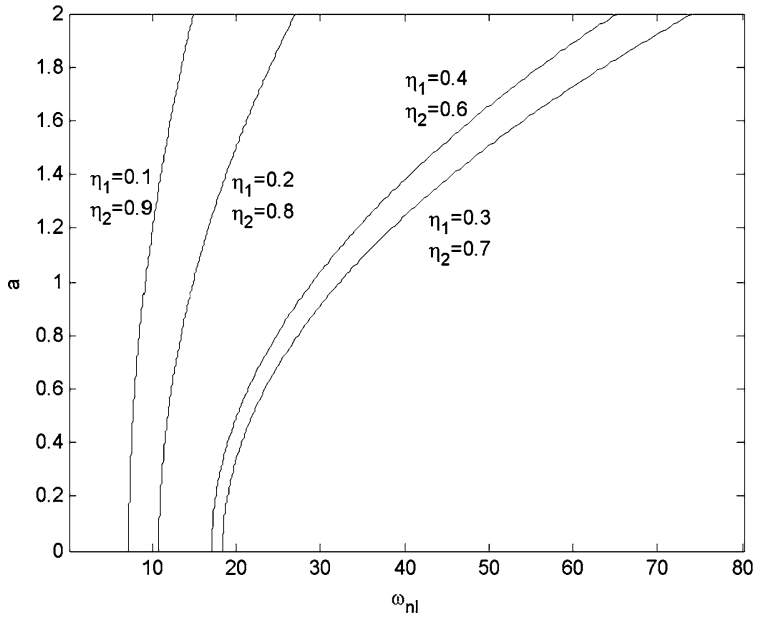

Fig. 18 Nonlinear frequency-amplitude variation for $v_{f}=0.2-v_{0}=0.2$ and different support locations (the first mode)

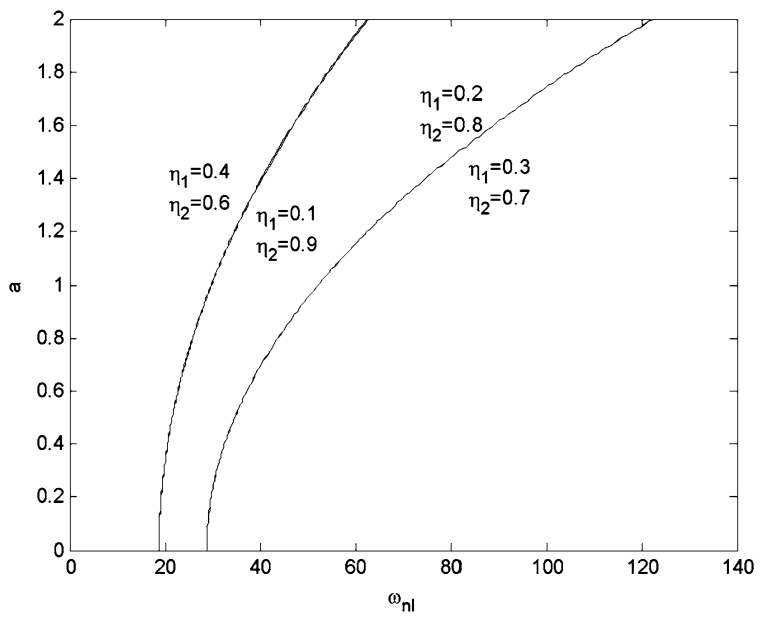

Fig. 19 Nonlinear frequency-amplitude variation for $v_{f}=0.2-v_{0}=0.2$ and different support locations (the second mode)

any number of supports. The stable and unstable solution regions are presented. An increase in axial mean speed decreases nonlinear frequencies. A more hardening type for higher velocities was observed. This is because of growing nonlinear corrections. Placing the intermediate supports around middle of the beam increases the corrections on the nonlinear frequencies. As the mean velocity increases, unstable regions widen for the same flexural values. Around zero-mean velocity, unstable regions are small; around critical velocity, it is wide. Increasing rigidity makes it narrow. The amplitudes of vibrations increase in the nontriv- 
ial solution regions. An increase in rigidity decreases nonlinear effects on the natural frequency. In com-

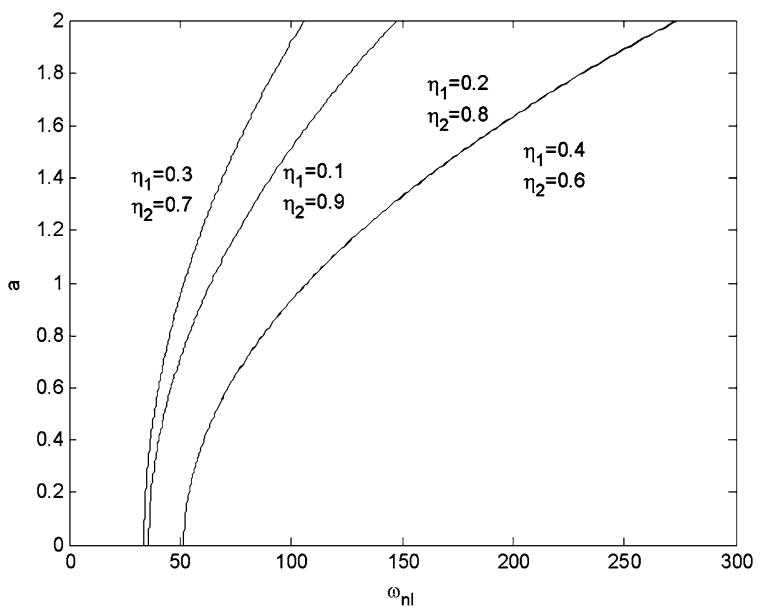

Fig. 20 Nonlinear frequency-amplitude variation for $v_{f}=0.2-v_{0}=0.2$ and different support locations (the third mode) bination resonances, amplitudes belonging to higher modes increase faster than those of lower modes. There is no increase in the amplitudes in difference types of combination resonances and no instability region in which trivial solution appears.

In Figs. 21 and 22, variation of combination resonances of sum type is presented for $v_{f}=0.2, \eta=0.1$, $v_{0}=0.2$, and two different frequency values $\left(\omega_{a}=\right.$ 5.0278, $\left.\omega_{b}=13.583\right)$. Solid lines denote stable regions, and dashed lines denote unstable regions. When velocity fluctuation frequency is close to the sum of the frequencies above, the behavior is more hardening for the upper mode amplitude.

Acknowledgements This work is supported by TUBITAK (The Scientific and Technological Research Council of Turkey) under project number MAG107M302.

Open Access This article is distributed under the terms of the Creative Commons Attribution License which permits any use, distribution, and reproduction in any medium, provided the original author(s) and the source are credited.
Fig. 21 Variation of amplitude with frequency parameter for combination resonances of sum type $\left(v_{f}=0.2, \eta=0.1\right.$, $v_{0}=0.2, \omega_{a}=5.028$, $\omega_{b}=13.583$ )

Fig. 22 Variation of amplitude with frequency parameter for combination resonances of sum type $\left(v_{f}=0.2, \eta=0.1\right.$ $v_{0}=0.2, \omega_{a}=5.028$, $\omega_{b}=13.583$ )
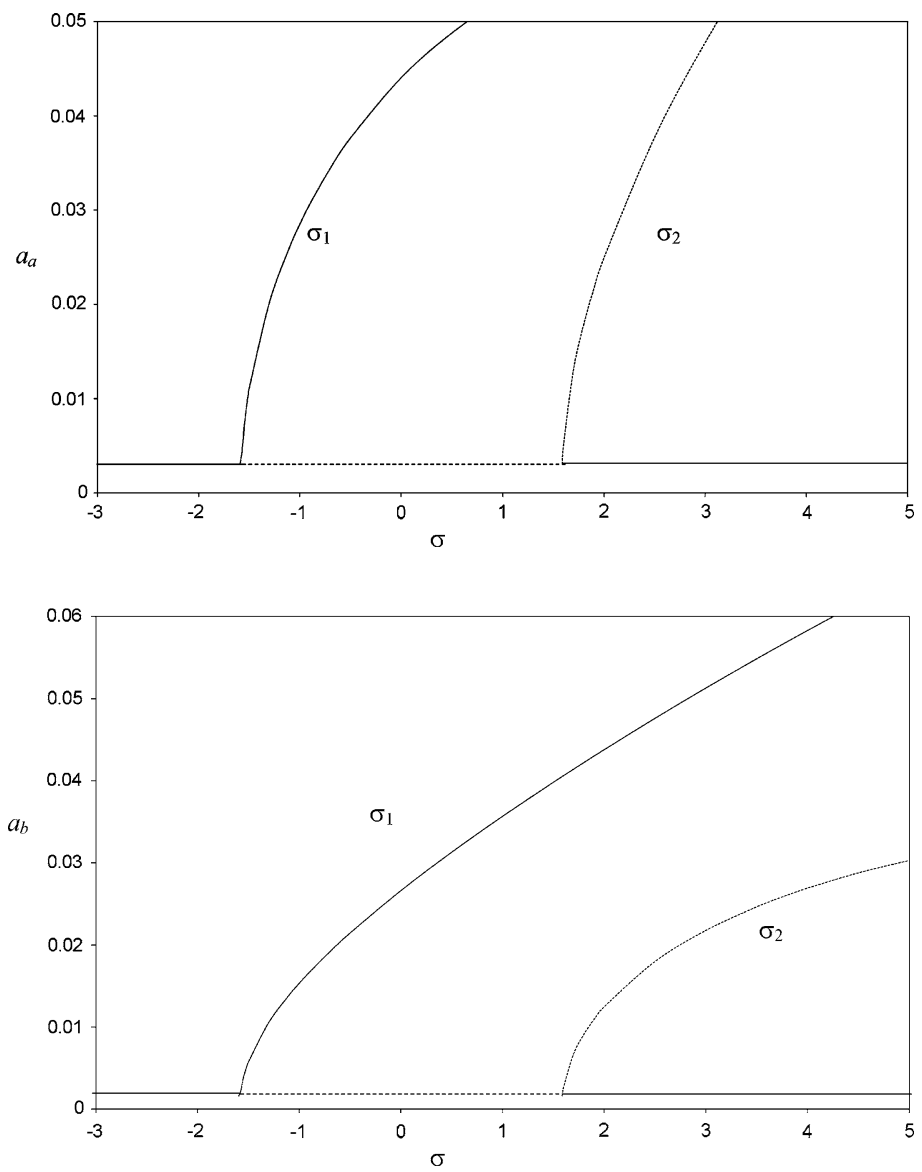


\section{Appendix}

$\beta_{n}=\beta_{n+4}$ for $n=1,2, \ldots, m$.

The \# of intermediate supports is given by $m=$ $0,1,2, \ldots, n$.

Size of the matrix $4(n+1) \times 4(n+1)$,

$m=0,1,2, \ldots, n$.

By defining the following matrices we can write the frequency matrix for arbitrary number of supports:

$r_{1}=\left[\begin{array}{cccc}1 & 1 & 1 & 1 \\ \beta_{1}^{2} & \beta_{2}^{2} & \beta_{3}^{2} & \beta_{4}^{2} \\ 0 & 0 & 0 & 0 \\ 0 & 0 & 0 & 0\end{array}\right]$

$r_{2}=\left[\begin{array}{cccc}0 & 0 & 0 & 0 \\ 0 & 0 & 0 & 0 \\ e^{i \beta_{1}} & e^{i \beta_{2}} & e^{i \beta_{3}} & e^{i \beta_{4}} \\ \beta_{1}^{2} e^{i \beta_{1}} & \beta_{2}^{2} e^{i \beta_{2}} & \beta_{3}^{2} e^{i \beta_{3}} & \beta_{4}^{2} e^{i \beta_{4}}\end{array}\right]$,

$r_{3}=\left[\begin{array}{cccc}e^{i \beta_{1} \eta_{n}} & e^{i \beta_{2} \eta_{n}} & e^{i \beta_{3} \eta_{n}} & e^{i \beta_{4} \eta_{n}} \\ 0 & 0 & 0 & 0 \\ \beta_{1} e^{i \beta_{1} \eta_{n}} & \beta_{2} e^{i \beta_{2} \eta_{n}} & \beta_{3} e^{i \beta_{3} \eta_{n}} & \beta_{4} e^{i \beta_{4} \eta_{n}} \\ -\beta_{1}^{2} e^{i \beta_{1} \eta_{n}} & -\beta_{2}^{2} e^{i \beta_{2} \eta_{n}} & -\beta_{3}^{2} e^{i \beta_{3} \eta_{n}} & -\beta_{4}^{2} e^{i \beta_{4} \eta_{n}}\end{array}\right]$, $r_{4}=\left[\begin{array}{cccc}0 & 0 & 0 & 0 \\ e^{i \beta_{1} \eta_{n}} & e^{i \beta_{2} \eta_{n}} & e^{i \beta_{3} \eta_{n}} & e^{i \beta_{4} \eta_{n}} \\ -\beta_{1} e^{i \beta_{1} \eta_{n}} & -\beta_{2} e^{i \beta_{2} \eta_{n}} & -\beta_{3} e^{i \beta_{3} \eta_{n}} & -\beta_{4} e^{i \beta_{4} \eta_{n}} \\ \beta_{1}^{2} e^{i \beta_{1} \eta_{n}} & \beta_{2}^{2} e^{i \beta_{2} \eta_{n}} & \beta_{3}^{2} e^{i \beta_{3} \eta_{n}} & \beta_{4}^{2} e^{i \beta_{4} \eta_{n}}\end{array}\right]$,

$r_{0}=\left[\begin{array}{llll}0 & 0 & 0 & 0 \\ 0 & 0 & 0 & 0 \\ 0 & 0 & 0 & 0 \\ 0 & 0 & 0 & 0\end{array}\right]$

For the three-support case (one intermediate support) 8 by 8 matrix, $1, \eta_{n}=1$ :

$r=\left[\begin{array}{cc}r_{1} & r_{2} \\ r_{3, n=1} & r_{4, n=1}\end{array}\right]$.

For the four-support case (two intermediate supports), 12 by 12 matrix:

$r=\left[\begin{array}{ccc}r_{1} & r_{0} & r_{2} \\ r_{3, n=1} & r_{4, n=1} & r_{0} \\ r_{0} & r_{3, n=2} & r_{4, n=2}\end{array}\right]$.

For the $(m+2)$-support case ( $m$ intermediate supports $), \quad 4(m+1)$ by $4(m+1)$ matrix:

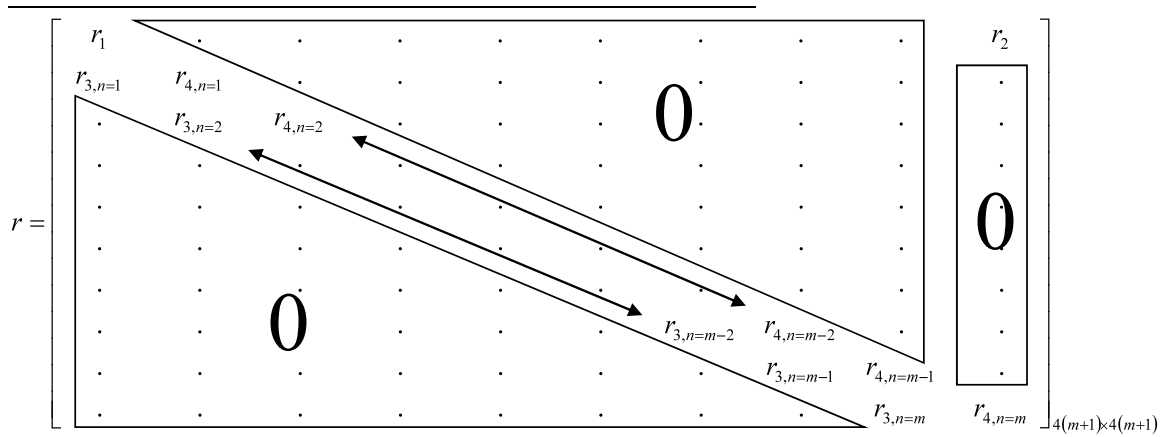

\section{References}

1. Ulsoy, A.G., Mote, C.D. Jr., Syzmani, R.: Principal developments in band saw vibration and stability research. Holz Roh- Werkst. 36, 273-280 (1978)

2. Wickert, J.A., Mote, C.D. Jr.: Current research on the vibration and stability of axially moving materials. Shock Vib. Dig. 20(5), 3-13 (1988)
3. Wickert, J.A., Mote, C.D. Jr.: Classical vibration analysis of axially moving continua. J. Appl. Mech. 57, 738-744 (1990)

4. Wickert, J.A.: Non-linear vibration of a traveling tensioned beam. Int. J. Non-Linear Mech. 27, 503-517 (1992)

5. Pakdemirli, M., Ulsoy, A.G.: Stability analysis of an axially accelerating string. J. Sound Vib. 203, 815-832 (1997)

6. Nayfeh, A.H., Nayfeh, J.F., Mook, D.T.: On methods for continuous systems with quadratic and cubic nonlinearities. Nonlinear Dyn. 3, 145-162 (1992) 
7. Öz, H.R., Pakdemirli, M., Özkaya, E.: Transition behaviour from string to beam for an axially accelerating material. J. Sound Vib. 215(3), 571-576 (1998)

8. Özkaya, E., Pakdemirli, M.: Vibrations of an axially accelerating beam with small flexural stiffness. J. Sound Vib. 234(3), 521-535 (2000)

9. Kural, S., Özkaya, E.: Vibrations of an axially accelerating, multiple supported flexible beam. Struct. Eng. Mech. 44(4), 521-538 (2012)

10. Pellicano, F., Zirilli, F.: Boundary layers and non-linear vibrations in an axially moving beam. Int. J. Non-Linear Mech. 33, 691-711 (1998)

11. Pakdemirli, M., Özkaya, E.: Approximate boundary layer solution of a moving beam problem. Math. Comput. Appl. 2(3), 93-100 (1998)

12. Yurddaş, A., Özkaya, E., Boyacı, H.: Nonlinear vibrations and stability analysis of axially moving strings having nonideal mid-support conditions. J. Vib. Control (2012). doi: $10.1177 / 1077546312463760$

13. Yurddaş, A., Özkaya, E., Boyacı, H.: Nonlinear vibrations of axially moving multi-supported strings having non-ideal support condition. Nonlinear Dyn. (2012). doi:10.1007/s11071-012-0650-5

14. Öz, H.R., Pakdemirli, M.: Vibrations of an axially moving beam with time-dependent velocity. J. Sound Vib. 227(2), 239-257 (1999)

15. Öz, H.R.: On the vibrations of an axially travelling beam on fixed supports with variable velocity. J. Sound Vib. 239(3), 556-564 (2001)

16. Özkaya, E., Öz, H.R.: Determination of stability regions of axially moving beams using artificial neural networks method. J. Sound Vib. 252(4), 782-789 (2002)

17. Bagdatli, S.M., Özkaya, E., Ozyigit, H.A., Tekin, A.: Nonlinear vibrations of stepped beam systems using artificial neural networks. Struct. Eng. Mech. 33(1), 15-30 (2009)

18. Öz, H.R., Pakdemirli, M., Boyac1, H.: Non-linear vibrations and stability of an axially moving beam with time dependent velocity. Int. J. Non-Linear Mech. 36, 107-115 (2001)

19. Öz, H.R.: Non-linear vibrations and stability analysis of tensioned pipes conveying fluid with variable velocity. Int. J. Non-Linear Mech. 36, 1031-1039 (2001)

20. Pakdemirli, M., Öz, H.R.: Infinite mode analysis and truncation to resonant modes of axially accelerated beam vibrations. J. Sound Vib. 311(3-5), 1052-1074 (2008)

21. Chen, L.Q., Yang, X.D.: Steady-state response of axially moving viscoelastic beams with pulsating speed: compar- ison of two nonlinear models. Int. J. Solids Struct. 42(1), 37-50 (2005)

22. Chen, L.Q., Yang, X.D.: Stability in parametric resonance of axially moving viscoelastic beams with time-dependent speed. J. Sound Vib. 284, 879-891 (2005)

23. Yang, X.D., Chen, L.Q.: Bifurcation and chaos of an axially accelerating viscoelastic beam. Chaos Solitons Fractals 23(1), 249-258 (2005)

24. Chen, L.Q., Yang, X.D.: Vibration and stability of an axially moving viscoelastic beam with hybrid supports. Eur. J. Mech. A, Solids 25, 996-1008 (2006)

25. Lewandowski, R.: Nonlinear free vibrations of multispan beams on elastic supports. Comput. Struct. 32(2), 305-312 (1989)

26. Gürgöze, M., Özgür, K., Erol, H.: On the eigenfrequencies of a cantilevered beam with a tip mass and in-span support. Comput. Struct. 56, 85-92 (1995)

27. Gürgöze, M., Erol, H.: Determination of the frequency response function of a cantilevered beam simply supported in-span. J. Sound Vib. 247, 372-378 (2001)

28. Özkaya, E., Bağdatli, S.M., Öz, H.R.: Non-linear transverse vibrations and 3:1 internal resonances of a beam with multiple supports. J. Vib. Acoust. (2008). doi:10.1115/ 1.2775508

29. Bağdatli, S.M., Öz, H.R., Özkaya, E.: Non-linear transverse vibrations and 3:1 internal resonances of a tensioned beam on multiple supports. J. Math. Comput. Appl. 16(1), 203215 (2011)

30. Bağdatli, S.M., Öz, H.R., Özkaya, E.: Dynamics of axially accelerating beams with an intermediate support. J. Vib. Acoust. (2011). doi:10.1115/1.4003205

31. Tekin, A., Özkaya, E., Bağdatli, S.M.: Three-to one internal resonances in multi stepped beam systems. Appl. Math. Mech. (2009). doi:10.1007/s10483-009-0907-x

32. Nayfeh, A.H.: Introduction to Perturbation Techniques. Wiley, New York (1981)

33. Nayfeh, A.H., Mook, D.T.: Nonlinear Oscillations. Wiley, New York (1979)

34. Ghayesh, M.H.: Coupled longitudinal-transverse dynamics of an axially accelerating beam. J. Sound Vib. 331(23), 5107-5124 (2012). doi:10.1016/j.jsv.2012.06.018

35. Ghayesh, M.H., Amabili, M., Païdoussis, M.P.: Nonlinear vibrations and stability of an axially moving beam with an intermediate spring support: twodimensional analysis. Nonlinear Dyn. 70(1), 335-354 (2012). doi:10.1007/s11071-012-0458-3 